\title{
THE SEMISIMPLE SUBALGEBRAS OF EXCEPTIONAL LIE ALGEBRAS
}

\author{
A. N. MINCHENKO
}

\begin{abstract}
Dynkin classified the maximal semisimple subalgebras of exceptional Lie algebras up to conjugacy, but only classified the simple subalgebras up to the coarser relation of linear conjugacy. In the present paper the simple subalgebras of exceptional Lie algebras are classified up to conjugacy, and their normalizers in the group are found. In a certain sense, this completes the description of the semisimple subalgebras of semisimple Lie algebras. As a by-product we obtain a list of all those semisimple subalgebras of exceptional Lie algebras for which the linear conjugacy class does not coincide with their conjugacy class (in the classical case the corresponding result was known).
\end{abstract}

\section{INTRODUCTION}

In what follows as a rule we shall assume that all the Lie algebras under consideration are complex semisimple. However, in certain cases it is more convenient to assume only reductivity, in which case this will be specifically stipulated.

Let $\mathfrak{g}$ be a semisimple Lie algebra. Two reductive subalgebras $\mathfrak{h}_{1}, \mathfrak{h}_{2}$ of $\mathfrak{g}$ are said to be linearly conjugate $\left(\mathfrak{h}_{1} \stackrel{L}{\sim} \mathfrak{h}_{2}\right)$ if for any finite-dimensional linear representation of the algebra $\mathfrak{g}$ these subalgebras become conjugate subalgebras of the matrix algebra. Clearly, conjugate subalgebras are linearly conjugate. The converse is false: several examples were given in [5]. Dynkin's idea was to divide the classification of subalgebras into two stages, the first of which is the classification up to linear conjugacy and the second is the analysis of the partitions of the linear conjugacy classes into conjugacy classes. This approach to the description of subalgebras is motivated by the fact that in order to determine whether two subalgebras are linearly conjugate it is sufficient to consider merely one or two irreducible representations of the algebra $\mathfrak{g}$ (see Theorem 2). Furthermore, linearly conjugate subalgebras are, as a rule, conjugate. This fact was pointed out in [5].

The following two results of Dynkin are most important for us. One of them is the enumeration of all the regular subalgebras ([5, Table 11]) and S-subalgebras ([5, Table 39]) of exceptional Lie algebras up to conjugacy, and the second is the enumeration of all the simple subalgebras of exceptional Lie algebras up to linear conjugacy ([5, Table 25]).

In the next section we give the basic definitions and assertions, which we will use extensively in what follows. In Section 2 we obtain a classification of the simple subalgebras of exceptional Lie algebras up to conjugacy and find their realizable outer automorphisms. An automorphism $\sigma$ of a subalgebra $\mathfrak{h} \subset \mathfrak{g}$ is said to be realizable in $\mathfrak{g}$ if there exists an inner automorphism $\theta$ of the algebra $\mathfrak{g}$ such that $\theta(\mathfrak{h})=\mathfrak{h}$ and $\left.\theta\right|_{\mathfrak{h}}=\sigma$. In Sections 3 and 4 we describe the semisimple subalgebras of exceptional Lie algebras whose linear conjugacy class is non-trivially partitioned into conjugacy classes, and indicate exactly

2000 Mathematics Subject Classification. Primary 17B25; Secondary 17B20, 22E10.

Key words and phrases. Exceptional Lie algebras, semisimple subalgebra, adjoint group, simple subalgebra, semisimple embedding, simple embedding, conjugacy class, normalizer. 
how this partition takes place. In Section 5, using the results of [1], we find the normalizers of all the connected simple subgroups of rank greater than one in exceptional Lie groups (the centralizers of the $\mathfrak{s l}_{2}$-subalgebras were found in the aforementioned paper). All the main results are presented in the tables at the end of the paper. These tables are described in the course of the exposition.

We point out that in this paper we correct several inaccuracies that are present in [5]. These will be indicated in the course of the exposition.

After this study was completed, the author discovered [9], in which some of the results of the present paper were obtained; namely, the classification up to conjugacy of the simple subalgebras of exceptional Lie algebras (over algebraically closed fields with minor restrictions on the characteristic), and their centralizers were also found. The results of the present paper agree with those of 9 .

The author is grateful to E. B. Vinberg for his interest in this work and his valuable comments on the preliminary version of this paper. The author is also grateful to I. V. Losev for useful discussions of certain questions.

\section{LIST OF NOTATION}

$\mathfrak{g}=\operatorname{Lie} G$ is the Lie algebra of a Lie group $G$; in what follows, unless otherwise stated, we assume $G$ to be the adjoint group;

$\widehat{G}$ is the simply connected covering of a connected Lie group $G$;

$G^{\circ}$ is the connected component of the identity of a group $G$;

$\mathrm{T}_{\mathrm{n}}, T_{n}$ are an $n$-dimensional algebraic torus and its tangent algebra (which we also call a torus if this causes no ambiguity);

Aut $\mathfrak{g}$ is the group of all automorphisms of an algebra $\mathfrak{g}$;

Int $\mathfrak{g}$ is the group of inner automorphisms of an algebra $\mathfrak{g}$;

$\mathfrak{z}(\mathfrak{g})$ is the center of an algebra $\mathfrak{g}$;

$\mathfrak{z}_{\mathfrak{g}}(\mathfrak{h})$ is the centralizer of a subalgebra $\mathfrak{h}$ in $\mathfrak{g}$;

$\mathfrak{n}_{\mathfrak{g}}(\mathfrak{h})=\mathfrak{h}+\mathfrak{z}_{\mathfrak{g}}(\mathfrak{h})$ is the normalizer of a subalgebra $\mathfrak{h}$ in $\mathfrak{g}$;

$Z_{G}(\mathfrak{h})$ is the centralizer of a subalgebra $\mathfrak{h} \subset \mathfrak{g}$ in the group $G$;

$\mathfrak{z} \mathfrak{g}(H)$ is the subalgebra of the algebra $\mathfrak{g}$ consisting of the fixed vectors under the adjoint action of a subgroup $H \subset G$;

$\mathfrak{g}^{\sigma}$ is the subalgebra of the algebra $\mathfrak{g}$ centralized by an automorphism $\sigma \in$ Aut $\mathfrak{g}$;

$N_{G}(\mathfrak{h})$ is the normalizer of a subalgebra $\mathfrak{h} \subset \mathfrak{g}$ in the group $G$;

$\Pi(\mathfrak{g})=\left\{\alpha_{1}, \ldots, \alpha_{n}\right\}$ is a system of simple roots of a simple Lie algebra $\mathfrak{g}$ of rank $n$; the numbering of simple roots is the same as in [3];

$\pi_{1}, \ldots, \pi_{n}$ are the fundamental weights corresponding to $\alpha_{1}, \ldots, \alpha_{n}$;

$R(\lambda)$ is the representation of the Lie algebra $\mathfrak{g}$ with highest weight $\lambda$;

$P \wedge Q$ is a semidirect product of groups $P$ and $Q$, where $Q$ is a normal factor;

$\mathbb{Z}_{n}$ is a cyclic group of order $n$;

$\mathbb{S}_{n}$ is the group of all permutations on $n$ elements;

$\mathbb{A}_{n}$ is the group of even permutations on $n$ elements;

$\mathbb{V}_{4}$ is a group isomorphic to $\mathbb{Z}_{2} \times \mathbb{Z}_{2}$ or, as a subgroup of $\mathbb{S}_{4}$, the Klein four-group;

Id is the identity transformation.

\section{Preliminaries}

1.1. Equivalence and linear equivalence. Embeddings $\varphi_{i}: \mathfrak{h} \hookrightarrow \mathfrak{g}, i=1,2$, are said to be equivalent $\left(\varphi_{1} \sim \varphi_{2}\right)$ if there exists an element $\theta \in \operatorname{Int} \mathfrak{g}$ such that $\varphi_{2}=\theta \circ \varphi_{1}$. It is easy to obtain a classification of subalgebras from a classification of embeddings: one must join those equivalence classes of embeddings $\mathfrak{h} \hookrightarrow \mathfrak{g}$ that are taken to one another by an outer automorphism of the algebra $\mathfrak{h}$ (and consider their images in $\mathfrak{g}$ ). Conversely, 
having a description of subalgebras and knowing which of the outer automorphisms are realized in $\mathfrak{g}$ one can obtain a classification of embeddings.

By analogy with the notion of linear conjugacy of subalgebras we have the notion of linear equivalence of embeddings. Namely, embeddings $\varphi_{i}: \mathfrak{h} \hookrightarrow \mathfrak{g}, i=1,2$, are linearly equivalent $\left(\varphi_{1} \stackrel{L}{\sim} \varphi_{2}\right)$ if for any representation $\rho: \mathfrak{g} \rightarrow \mathfrak{g l}(V)$ the corresponding representations $\rho \circ \varphi_{i}, i=1,2$, of the algebra $\mathfrak{h}$ are isomorphic. Obviously, equivalence implies linear equivalence. The connection between equivalence and linear equivalence is described by the following theorem (see [5, Theorem 1.1]).

Theorem 1. Two embeddings $\varphi_{i}: \mathfrak{h} \hookrightarrow \mathfrak{g}, i=1,2$, are linearly equivalent if and only if their restrictions to a Cartan subalgebra of the algebra $\mathfrak{h}$ are equivalent.

Corollary 1. Two isomorphic subalgebras $\mathfrak{h}_{1}, \mathfrak{h}_{2}$ of an algebra $\mathfrak{g}$ are linearly conjugate if and only if some of their dual systems of simple roots are conjugate in $\mathfrak{g}$ (duality is interpreted in the sense of the Cartan-Killing form).

Proof. In one direction the assertion obviously follows from Theorem 1. We now prove that the linear conjugacy implies the conjugacy of two systems of dual simple roots. In view of Theorem 1 for that it is sufficient to prove that there exists a representation $\psi$ of the algebra $\mathfrak{g}$ such that the linear equivalence of an arbitrary pair of embeddings $\varphi_{i}: \mathfrak{h} \hookrightarrow \mathfrak{g}, i=1,2$, is determined by the equivalence of the representations $\psi \circ \varphi_{1}$ and $\psi \circ \varphi_{2}$ of the algebra $\mathfrak{h}$.

Let $\psi_{1}, \ldots, \psi_{n}$ be representations of the algebra $\mathfrak{g}$ whose highest weights generate the space $\mathfrak{t}^{*}$, where $\mathfrak{t}$ is a Cartan subalgebra of $\mathfrak{g}$. It is known [5, Theorem 1.2] that then the linear equivalence of a pair of embeddings follows immediately from their equivalence with respect to the representations $\psi_{1}, \ldots, \psi_{n}$.

Let $\varphi: \mathfrak{h} \hookrightarrow \mathfrak{g}$ be an arbitrary embedding, $\mathcal{M}$ the set of all irreducible representations of the algebra $\mathfrak{h}$, and $z=\left(k_{1}, \ldots, k_{n}\right) \in \mathbb{N}^{n}$ an $n$-tuple of positive integers. We consider the composite of maps

$$
\mathcal{M} \stackrel{\alpha}{\longrightarrow} \mathbb{Z}^{n} \stackrel{\beta_{z}}{\longrightarrow} \mathbb{Z}
$$

where $\alpha(x)=\left(m_{1}(x), m_{2}(x), \ldots, m_{n}(x)\right)$ is the $n$-tuple of multiplicities of a representation $x$ in $\psi_{i} \circ \varphi$ and $\beta_{z}(w)=(z, w)$ is the ordinary scalar product. The set $\operatorname{Im} \alpha$ is in any case contained in some bounded parallelepiped (independent of $\varphi$ ) and is consequently finite. Therefore there exists a point $z \in \mathbb{N}^{n}$ such that the map $\beta_{z}$ is one-to-one on $\operatorname{Im} \alpha$. We claim that then the corresponding representation $\psi=\sum_{i=1}^{n} k_{i} \psi_{i}$ is the required one. Indeed, $\beta_{z}(\alpha(x))$ is the multiplicity of an irreducible representation $x$ in $\psi \circ \varphi$. By the choice of $z$, this multiplicity uniquely determines $\alpha(x)$, as required.

Remark 1. In [5] this corollary (Theorem 1.5) is stated without proof and we deemed it appropriate to fill this gap. We also point out that the existence of the representation $\psi$ in the proof of the corollary was proved in [8, albeit by a somewhat different method.

In particular, the linear conjugacy classes of subalgebras $\mathfrak{h} \subset \mathfrak{g}$ are obtained by joining the linear equivalence classes of embeddings $\mathfrak{h} \hookrightarrow \mathfrak{g}$ that are taken to each other by outer automorphisms of the algebra $\mathfrak{h}$. Conversely, a classification of embeddings can be obviously obtained from a classification of subalgebras if it is known which outer automorphisms of dual systems of simple roots of the algebras $\mathfrak{h}$ are realized in $\mathfrak{g}$ (or, which is the same, in the Weyl group of the algebra $\mathfrak{g}$ ).

In the present paper we actually solve the problem of classifification of embeddings into exceptional Lie algebras up to equivalence. As we have already mentioned, this is a more general problem than its analogue for subalgebras. By Theorem 7 , which we prove in Section 4, the classification of embeddings up to equivalence reduces to their 
classification up to linear equivalence. The latter is substantially simplified by using the following assertion 1

Theorem 2. Let $\mathfrak{g}$ be a simple Lie algebra, and $\varphi_{i}: \mathfrak{h} \hookrightarrow \mathfrak{g}, i=1,2$, two embeddings.

1) If $\mathfrak{g} \neq \mathfrak{s o}_{2 n}$, then $\varphi_{1} \stackrel{L}{\sim} \varphi_{2} \Longleftrightarrow \pi_{1} \circ \varphi_{1} \sim \pi_{1} \circ \varphi_{2}$;

2) if $\mathfrak{g}=\mathfrak{s o}_{2 n}$, then $\varphi_{1} \stackrel{L}{\sim} \varphi_{2} \Longleftrightarrow \pi_{k} \circ \varphi_{1} \sim \pi_{k} \circ \varphi_{2}, k=1, \ldots, n$,

where $\pi_{i}$ is the fundamental representation corresponding to the $i$-th simple root.

Remark 2. In a weaker version, this theorem was stated in [5, Theorem 1.3]. Dynkin proved that to verify the linear equivalence it is sufficient to consider those sets of representations of the algebra $\mathfrak{g}$ for which the set of weights belonging to the levels of width 1 generates the space $\mathfrak{t}^{*}$. Therefore a single representation was not enough for the cases $\mathfrak{g}=E_{7}, E_{8}$ : in addition it was necessary to look at the representations $\pi_{6}$, $\pi_{7}$, respectively 2 Dynkin's result was strengthened in [8], using fundamentally different considerations.

We now discuss in detail the problem of the classification of embeddings $\varphi: \mathfrak{h} \hookrightarrow \mathfrak{g}$ up to linear equivalence. This problem appears to be fairly manageable. Indeed, if $\mathfrak{g}$ is a classical Lie algebra, then the answer is given by Theorem 2, Next, in [5] a classification of the maximal subalgebras of exceptional Lie algebras $\mathfrak{g}$ up to conjugacy was obtained and the restrictions to them of the simplest $\left(\pi_{\mathfrak{g}}\right)$ and adjoint $\left(\operatorname{ad}_{\mathfrak{g}}\right)$ representations of the algebra $\mathfrak{g}$ were found (see [5, Tables 25, 35], [10]); we point out that the restrictions of the representations $\operatorname{ad}_{\mathfrak{g}}$ to the maximal regular subalgebras can easily be obtained by the method expounded in 2]). Let $\varphi(\mathfrak{h}) \subset \mathfrak{m} \subset \mathfrak{g}$, where $\mathfrak{m}$ is a maximal subalgebra of $\mathfrak{g}$. By what we have said above, we can reduce the question to the case where $\mathfrak{m}$ is a sum of classical algebras.

Recall the simplest representations of exceptional Lie algebras:

$$
G_{2} \subset \mathfrak{s o}_{7}, \quad F_{4} \subset \mathfrak{s o}_{26}, \quad E_{6} \subset \mathfrak{s l}_{27}, \quad E_{7} \subset \mathfrak{s p}_{56}, \quad E_{8} \subset \mathfrak{s o}_{248} .
$$

Note that $\pi_{E_{8}} \sim \operatorname{ad}_{E_{8}}$. It is known that we have the chain of inclusions

$$
G_{2} \subset F_{4} \subset E_{6} \subset E_{7} \subset E_{8}
$$

and all such chains are conjugate in $E_{8}$. In addition, the following hold:

$$
\begin{aligned}
& \left.\pi_{F_{4}}\right|_{G_{2}} \sim 3 \pi_{G_{2}}+5 N, \\
& \left.\pi_{E_{6}}\right|_{F_{4}} \sim \pi_{F_{4}}+N, \\
& \left.\pi_{E_{7}}\right|_{E_{6}} \sim \pi_{E_{6}}+\pi_{E_{6}}^{*}+2 N, \\
& \left.\pi_{E_{8}}\right|_{E_{7}} \sim \operatorname{ad}_{E_{7}}+\pi_{E_{7}}+3 N,
\end{aligned}
$$

where $N$ is the trivial representation and $R^{*}$ is the conjugate representation of $R$.

Thus, verifying the linear equivalence of embeddings into the exceptional Lie algebras reduces to calculating the restrictions to them of representations of the classical Lie algebras (although in many cases even this is not needed by virtue of the tables in [5, 10, ). We do not carry out a more detailed investigation, since this is not required for the main thrust of this paper.

We now present known facts, which reduce the classification of embeddings up to equivalence to the classification up to linear equivalence, in certain cases. As above, let $\varphi_{i}: \mathfrak{h} \hookrightarrow \mathfrak{g}, i=1,2$.

\footnotetext{
${ }^{1}$ In what follows we assume that $n \geq 4$ for the algebra $\mathfrak{s o}_{2 n}$.

${ }^{2}$ The remark after Theorem 11.2 in [5] relates to simple subalgebras.
} 
Theorem 3. We have $\varphi_{1} \sim \varphi_{2} \Longleftrightarrow \varphi_{1} \stackrel{L}{\sim} \varphi_{2}$ under any of the following conditions:

1. $\mathfrak{h} \simeq \mathfrak{s l}_{2} ;$

2. $\operatorname{Im} \varphi_{i}, i=1,2$, are regular subalgebras;

3. $\mathfrak{g}=\mathfrak{s l}_{n}, \mathfrak{s p}_{n}, \mathfrak{s o}_{2 n+1}, G_{2}, F_{4}$.

We comment on the assertions of the theorem. Assertion 1 can be formulated as follows: two $\mathfrak{s l}_{2}$-subalgebras are conjugate if and only if their Dynkin characteristics are conjugate. Apparently, this assertion was first proved by Mal'tsev (see 7]). We shall discuss assertion 2 in the next subsection. When $\mathfrak{g}=\mathfrak{s l}_{n}$, assertion 3 is the definition of an isomorphism of representations. The proof for the algebras $\mathfrak{g}=\mathfrak{s p}_{n}, \mathfrak{s o}_{2 n+1}$ was given in 7 (in the last case the proof follows from a fact whose proof goes back to Frobenius: Equivalent completely reducible orthogonal representations are orthogonally equivalent). The case $\mathfrak{g}=G_{2}$ is obvious in view of parts 1 and 2 . The case $\mathfrak{g}=F_{4}$ was analyzed in [8].

Theorem 4. Suppose that $\mathfrak{g}=\mathfrak{s o}_{2 n}$ in the preceding notation.

1. If the embeddings $\varphi_{1}, \varphi_{2}$ are linearly equivalent but not equivalent, then $\varphi_{2}=$ $\sigma \circ \varphi_{1}$, where $\sigma$ is an outer automorphism of the algebra $\mathfrak{g}$ defined by an element of the orthogonal group $\mathrm{O}_{2 \mathrm{n}}$.

2. The embeddings $\varphi, \sigma \circ \varphi$ are linearly equivalent if and only if the representation $\pi_{1} \circ \varphi$ of the algebra $\mathfrak{h}$ contains the zero weight.

3. The embeddings $\varphi, \sigma \circ \varphi$ are equivalent if and only if the representation $\pi_{1} \circ \varphi$ has an odd-dimensional orthogonal subrepresentation.

Parts 1 and 3 of the theorem were proved in [7, and part 2 in [5, Theorem 1.4].

Example 1. The adjoint representations of the algebras $\mathfrak{s l}_{3}$ and $\mathfrak{s o}_{5}$ determine their embeddings into the algebras $\mathfrak{s o}_{8}$ and $\mathfrak{s o}_{10}$, respectively. Applying Theorem 4 one can easily see that the linear equivalence classes of these embeddings consist of two classes of equivalent embeddings and that these are the only embeddings into these algebras with this property. We point out that for embeddings $\mathfrak{h} \hookrightarrow \mathfrak{s o}_{2 n}$, where $\mathfrak{h}$ is a direct sum of algebras isomorphic to $\mathfrak{s l}_{2}$, the notions of equivalence and linear equivalence coincide, since an irreducible even-dimensional representation of such an algebra $\mathfrak{h}$ cannot have zero weight.

Thus, it remains to examine the cases $\mathfrak{g}=E_{6}, E_{7}$, and $E_{8}$. The corresponding result is contained in Theorem 7 . Namely, we enumerate all the cases where a class of linearly equivalent embeddings into $\mathfrak{g}$ is non-trivially partitioned into classes of equivalent embeddings, and it is also indicated exactly how this partition takes place. There are 3 such cases for the algebra $E_{6}, 2$ for $E_{7}$, and 10 for $E_{8}$, and in each case the partition is into exactly two equivalence classes. A similar assertion (Corollary 3) is proved for subalgebras. We point out that the methods of dealing with simple and non-simple semisimple embeddings are essentially different. In the first case we rely on Dynkin's classification of the simple embeddings, and in the second, on Theorems 3 and 4 .

1.2. Description of regular subalgebras. A reductive subalgebra $\mathfrak{h} \subset \mathfrak{g}$ is said to be regular if one of the following equivalent conditions holds:

(1) the subalgebra $\mathfrak{h}$ is normalized by some maximal torus $\mathfrak{t}$ of the algebra $\mathfrak{g}$;

(2) the reductive subalgebra $\mathfrak{n}_{\mathfrak{g}}(\mathfrak{h}) \subset \mathfrak{g}$ has maximal rank.

In particular, if a subalgebra $\mathfrak{h} \subset \mathfrak{g}$ is regular, then the relation $\mathfrak{n}_{\mathfrak{g}}(\mathfrak{h})=\mathfrak{h}+\mathfrak{z}_{\mathfrak{g}}(\mathfrak{h})$ implies that $\mathfrak{t}=\mathfrak{t} \cap \mathfrak{h}+\mathfrak{t} \cap \mathfrak{z}_{\mathfrak{g}}(\mathfrak{h})$. An embedding whose image is a regular subalgebra is called a regular embedding. If in these definitions we replace Lie algebras by Lie groups, then 
we obtain the definitions of a regular subgroup and a regular embedding. Note that subalgebras (subgroups) of maximal rank are regular.

We point out that it follows from the definition that up to equivalence, a regular embedding is uniquely determined by its restriction to a Cartan subalgebra (assertion 2 of Theorem 3) 3 Indeed, let $\varphi: \mathfrak{h} \hookrightarrow \mathfrak{g}$ be a regular embedding, and $\mathfrak{k} \subset \mathfrak{h}$ a Cartan subalgebra. We choose a maximal torus $\mathfrak{t} \subset \mathfrak{z}_{\mathfrak{g}}(\mathfrak{k}) \mathbb{4}$ Using conjugation by an element of $\mathfrak{z}_{\mathfrak{g}}(\mathfrak{k})$ we can make sure that the condition $\mathfrak{t} \subset \mathfrak{n}_{\mathfrak{g}}(\mathfrak{h})$ holds. Then the map $\varphi$ will take root subspaces to root subspaces, and to a root $\alpha \in \mathfrak{k}^{*}$ there will correspond a root of the algebra $\mathfrak{g}$ that is equal to 0 on $\mathfrak{t} \cap \mathfrak{z}_{\mathfrak{g}}(\varphi(\mathfrak{h}))$, and to $\left(\left.\varphi^{*}\right|_{\varphi(\mathfrak{k})}\right)^{-1}(\alpha)$ on $\varphi(\mathfrak{k})$. This argument, with the fact that maximal tori are conjugate in $\mathfrak{z}_{\mathfrak{g}}(\varphi(\mathfrak{k}))$, implies that linearly equivalent regular embeddings are equivalent.

Dynkin [5] set out a construction which lets us describe all the semisimple regular subalgebras of a given semisimple Lie algebra $\mathfrak{g}$. It is clearly sufficient for us to be able to find the maximal subalgebras among these subalgebras. Consequently, we can assume the algebra $\mathfrak{g}$ to be simple. We consider the subalgebras whose systems of simple roots are obtained by deleting one element either from the extended or the ordinary system of simple roots of the algebra $\mathfrak{g}$. Then every maximal regular subalgebra of $\mathfrak{g}$ is conjugate to one of the algebras obtained in this way. This description, in particular, implies the following.

Proposition 1. Every proper regular subalgebra has non-trivial centralizer in the group $G=$ Int $\mathfrak{g}$. The centralizer of a semisimple maximal subalgebra of maximal rank has prime order.

Corollary 2. Let $R \subset G$ be a connected reductive subgroup of maximal rank of a connected reductive Lie group $G$, and let $Z=Z_{G}(R)=Z(R)$ be its centralizer (which coincides with its center). Then $R=Z_{G}(Z)^{\circ}$.

Proof. We set $R^{\prime}=Z_{G}(Z)^{\circ}$. Then $R \subset R^{\prime}$ is a subgroup of maximal rank and $Z(R)=$ $Z\left(R^{\prime}\right)$. Taking the quotients of the groups $R$ and $R^{\prime}$ by the common center we use the first part of Proposition 1 to obtain that the resulting quotients coincide. But then $R=R^{\prime}$.

We might expect that when the rank rk $\mathfrak{g}$ is small, semisimple regular subalgebras of the same type will be conjugate. This is indeed the case: if $\mathfrak{g}$ is one of the exceptional Lie algebras, then there are only 6 exceptions for $\mathfrak{g}=E_{7}$, and 5 for $\mathfrak{g}=E_{8}$, and in each such case the set of regular subalgebras of the same type contains exactly two conjugacy classes. To be precise, one of these classes is distinguished by the fact that the system of simple roots of some representative of it is contained in $A_{7}$ (respectively, in $A_{8}$ ) in the case $\mathfrak{g}=E_{7}$ (respectively, $E_{8}$ ). If we denote regular subalgebras by the same symbols as their types (that is what we shall do in what follows), then to denote this class we shall use a prime, and for the other class, two primes. For example,

$$
A_{5}^{\prime \prime} \not \subset A_{7} \subset E_{7}, \quad\left(2 A_{3}\right)^{\prime} \subset A_{8} \subset E_{8} .
$$

We mention another nice property of regular subalgebras. Recall that a Weyl involution of a reductive Lie algebra is an automorphism acting as multiplication by -1 on some maximal torus of the algebra. Such automorphisms do exist, and they are all conjugate by inner automorphisms.

Proposition 2. Let $\mathfrak{h}$ be a regular subalgebra of a reductive Lie algebra $\mathfrak{g}$. Then there exists a Weyl involution $\theta$ of the algebra $\mathfrak{g}$ such that $\theta(\mathfrak{h})=\mathfrak{h}$ and $\left.\theta\right|_{\mathfrak{h}}$ is a Weyl involution of the algebra $\mathfrak{h}$.

\footnotetext{
${ }^{3}$ This assertion was also proved in [8].

${ }^{4}$ In what follows, for simplicity we shall often omit the symbol $\varphi$, for example, $\mathfrak{z} \mathfrak{g}(\mathfrak{k})=\mathfrak{z} \mathfrak{g}(\varphi(\mathfrak{k}))$.
} 
Proof. For $\theta$ one must take an involution acting by inversion on a torus normalizing the subalgebra $\mathfrak{h}$.

Remark 3. It is known that for a simple Lie algebra $\mathfrak{g}$ we have $\theta \notin \operatorname{Int} \mathfrak{g}$ only in the cases $\mathfrak{g}=\mathfrak{s l}_{n}, n \geq 3, \mathfrak{s o}_{4 n+2}$, and $E_{6}$.

1.3. Complete regular subalgebras. A regular subalgebra of a reductive Lie algebra $\mathfrak{g}$ is said to be complete if it is not contained as a proper subalgebra in any regular subalgebra of the same rank. Complete semisimple subalgebras are characterized by the fact that they are the derived subalgebras of the centralizers of tori, or by the fact that their system of simple roots can be supplemented to a system of simple roots of the algebra $\mathfrak{g}$. In what follows, the set of regular (respectively, complete regular) subalgebras containing a given subalgebra $\mathfrak{h} \subset \mathfrak{g}$ and considered up to conjugacy in $\mathfrak{g}$ will be denoted by $\mathcal{R}(\mathfrak{h})$ (respectively, $\widetilde{\mathcal{R}}(\mathfrak{h})$ ). Complete regular subalgebras are important for us for the following reason.

Proposition 3. Let $\mathfrak{h}$ be a semisimple subalgebra of a semisimple Lie algebra $\mathfrak{g}$.

1. There exists a unique subalgebra $\widetilde{\mathfrak{r}} \in \widetilde{\mathcal{R}}(\mathfrak{h})$ (up to conjugacy) such that

$$
\operatorname{rk} \widetilde{\mathfrak{r}}=\min _{\mathfrak{l} \in \widetilde{\mathcal{R}}(\mathfrak{h})} \operatorname{rkl} \text {. }
$$

2. If embeddings $\varphi_{i}: \mathfrak{h} \hookrightarrow \widetilde{\mathfrak{r}} \subset \mathfrak{g}, i=1,2$, are equivalent in $\mathfrak{g}$, then the element $\theta \in \operatorname{Int} \mathfrak{g}$ in the definition of equivalence can be chosen so that $\theta(\mathfrak{\mathfrak { r }})=\widetilde{\mathfrak{r}}$.

Proof. We choose a maximal torus $\mathfrak{k} \subset \mathfrak{z}_{\mathfrak{g}}(\mathfrak{h})$. Then for $\widetilde{\mathfrak{r}}$ one must take the derived subalgebra of the subalgebra $\mathfrak{z}_{\mathfrak{g}}(\mathfrak{k})$. The assertions of the proposition follow from the conjugacy of maximal tori in $\mathfrak{z}_{\mathfrak{g}}(\mathfrak{k})$.

1.4. R- and S-subalgebras. Let $\mathfrak{h}$ be a reductive subalgebra of a semisimple Lie algebra $\mathfrak{g}$ and let $G=\operatorname{Int} \mathfrak{g}$.

A subalgebra $\mathfrak{h}$ is called an $R$-subalgebra if $\mathcal{R}(\mathfrak{h}) \neq\{\mathfrak{g}\}$. Otherwise it is called an $S$ subalgebra. The algebra $\mathfrak{g}$ is regarded as an S-subalgebra of itself. Clearly, every proper regular subalgebra is an R-subalgebra, and the principal three-dimensional subalgebra is an S-subalgebra. In a similar fashion we define $\mathrm{R}$ - and S-embeddings, as well as $\mathrm{R}$ and S-subgroups. Note that an S-subalgebra is necessarily semisimple (otherwise it would be contained in the centralizer of a non-trivial torus). These subalgebras can be easily characterized.

Proposition 4. A connected subgroup $H \subset G=\operatorname{Int} \mathfrak{g}$ is an $S$-subgroup if and only if $Z_{G}(H)=\{e\}$.

Proof. The implication "if" follows immediately from Proposition 1, Now let $H$ be an S-subgroup of $G$. We take an arbitrary semisimple element $s \in Z_{G}(H)$. Then $Z_{G}(s)$ is a regular subgroup of $G$ containing $H$. This subgroup coincides with the whole group $G$ only in the case $s=e$.

We shall also be interested in the subalgebras that have trivial centralizers in the group Aut g. We call them T-subalgebras. Clearly, every T-subalgebra is an S-subalgebra. The converse is false. For example, the subalgebra $F_{4}$ of the algebra $E_{6}$ is an Sbut not a T-subalgebra. The same can be said about the diagonal inclusion $\mathfrak{h} \subset \mathfrak{h}+\mathfrak{h}$.

Proposition 5. Let $\varphi_{i}: \mathfrak{h} \hookrightarrow \mathfrak{r} \subset \mathfrak{g}, i=1,2$, be equivalent embeddings of semisimple Lie algebras that are T-embeddings into $\mathfrak{r}$ and suppose that $\mathcal{R}(\mathfrak{h})=\{\mathfrak{r}, \mathfrak{g}\}$. Then the element $\theta \in \operatorname{Int} \mathfrak{g}$ in the definition of equivalence normalizes $\mathfrak{r}$. 
Proof. We set $Z=Z_{G}(\mathfrak{r}) \simeq \mathbb{Z}_{p}$, where $p \in \mathbb{N}$ is prime (see Proposition 11). It is sufficient to show that $Z_{0} \doteqdot Z_{G}(\mathfrak{h})=Z$ (we identify the subalgebra $\mathfrak{h}$, for example, with $\varphi_{1}(\mathfrak{h})$ ). Note that the group $Z_{0}$ contains no elements of order distinct from $p$. Suppose that the order of the group $Z_{0}$ is not prime. Then there exists an element of $Z_{0}$ normalizing (and even centralizing) the subgroup $Z$ and not contained in it. Therefore this element acts non-trivially on the subalgebra $\mathfrak{r}$ and centralizes the subalgebra $\mathfrak{h}$. We arrive at a contradiction with the fact that $\mathfrak{h}$ is a T-subalgebra.

In [5] all the S-subalgebras of exceptional Lie algebras were classified up to conjugacy (but the non-regular R-subalgebras were not classified). The S-subalgebras of the exceptional Lie algebras are analogues of the irreducible subalgebras of the classical Lie algebras. Indeed, in the cases $\mathfrak{g}=\mathfrak{s l}_{n}, \mathfrak{s p}_{n}, \mathfrak{s o}_{2 n+1}$ the S-subalgebras are precisely the irreducible subalgebras. For $\mathfrak{g}=\mathfrak{s o}_{2 n}$ the class of S-subalgebras is somewhat wider: it includes in addition the subalgebras with respect to which the underlying vector space decomposes into a sum of two non-equivalent simple odd-dimensional submodules that are non-degenerate in the sense of the scalar product 5 In the last case the proof is based on the fact that the maximal regular subalgebras in $\mathfrak{s o}_{2 n}$ are $\mathfrak{s o}_{2 k}+\mathfrak{s o}_{2(n-k)}, k=2, \ldots, n-2$, and $\mathfrak{s l}_{n}$. It is interesting to note that every R-subalgebra for any faithful representation of the algebra $\mathfrak{g}$ is represented by a reducible system of matrices [5, Theorem 7.1].

Clearly, the projections of S-subalgebras onto ideals of the algebra $\mathfrak{g}$ are S-subalgebras of these ideals.

Example 2. Suppose that an algebra $\mathfrak{g}$ is the direct sum of two isomorphic ideals: $\mathfrak{g}=\mathfrak{h} \oplus \mathfrak{h}$. Then there is a one-to-one correspondence between the elements of the group Aut $\mathfrak{h} /$ Int $\mathfrak{h}$ and the conjugacy classes of the S-subalgebras isomorphic to $\mathfrak{h}$. Namely, these classes can be described by the following representatives:

$$
\mathfrak{h}_{i}=\left\{x+\sigma_{i}(x): x \in \mathfrak{h}\right\}
$$

where $\sigma_{i} \in$ Aut $\mathfrak{h}$ are representatives of the cosets of Int $\mathfrak{h}$ in Aut $\mathfrak{h}$. Clearly, the outer automorphisms of the subalgebras $\mathfrak{h}_{i}$ are not realized in $\mathfrak{g}$. Therefore, if we consider the embeddings $\mathfrak{h} \hookrightarrow \mathfrak{g}$, the number of their equivalence classes is given by the square of the number of conjugacy classes of the subalgebras of $\mathfrak{g}$ isomorphic to $\mathfrak{h}$.

This example can be obviously generalized to the case of an arbitrary number of isomorphic ideals whose sum gives a decomposition of an algebra $\mathfrak{g}$.

Let $\mathfrak{h}$ be a maximal S-subalgebra of a simple exceptional Lie algebra $\mathfrak{g}$. Then by Dynkin's result the algebra $\mathfrak{h}$ is isomorphic to one in the following list:

$$
\begin{array}{ll}
\mathfrak{g}=G_{2}: & \mathfrak{s l}_{2} ; \\
\mathfrak{g}=F_{4}: & \mathfrak{s l}_{2}, G_{2}+\mathfrak{s l}_{2} ; \\
\mathfrak{g}=E_{6}: & \mathfrak{s l}_{3}, G_{2}, \mathfrak{s p}_{8}, G_{2}+\mathfrak{s l}_{3}, F_{4} ; \\
\mathfrak{g}=E_{7}: & \mathfrak{s l}_{2}, \mathfrak{s l}_{2}+\mathfrak{s l}_{2}, \mathfrak{s l}_{3}, G_{2}+\mathfrak{s l}_{2}, G_{2}+\mathfrak{s p}_{6}, F_{4}+\mathfrak{s l}_{2} ; \\
\mathfrak{g}=E_{8}: & \mathfrak{s l}_{2}, \mathfrak{s o}_{5}, \mathfrak{s l}_{3}+\mathfrak{s l}_{2}, G_{2}+F_{4},
\end{array}
$$

and isomorphic subalgebras are conjugate, except for the cases $\mathfrak{s l}_{2} \subset E_{7}, E_{8}$ (where there are, respectively, two and three such subalgebras) and $A_{2}, G_{2} \subset E_{6}$, which are not conjugate to their images under an outer automorphism of $E_{6}$ (see [5, Theorem 11.1]).

\footnotetext{
${ }^{5}$ There is an inaccuracy in [5, Theorem 7.2]: the submodules do not have to be non-isomorphic.
} 
1.5. One property of linearly conjugate subalgebras. Let $\mathfrak{h}$ be a semisimple subalgebra of a semisimple Lie algebra $\mathfrak{g}$. In what follows it will be convenient to use the notation

$$
\mathcal{R}(\mathfrak{h})^{L}=\bigcup_{\substack{\mathfrak{h}^{\prime} \\ \sim}} \mathcal{R}\left(\mathfrak{h}^{\prime}\right) .
$$

We define the corank of the subalgebra $\mathfrak{h}$ to be the number cork $\mathfrak{h}=\operatorname{rk} \mathfrak{g}-\operatorname{rk} \mathfrak{h}$. For a non-negative integer $d$ we denote by $m(d)$ the minimum possible rank of a reductive Lie algebra of dimension $d$. We have the following obvious proposition.

Proposition 6. Let $d=\operatorname{dim}_{\mathfrak{z}}(\mathfrak{h})$. Then there exists a complete regular subalgebra containing $\mathfrak{h}$ of corank at least $m(d)$.

By considering the adjoint representation of the algebra $\mathfrak{g}$, we observe that the centralizers of linearly conjugate subalgebras must have equal dimensions. In this connection we note the following.

Proposition 7. Let $c, c^{\prime}$ be the maximum possible coranks of subalgebras in $\mathcal{R}(\mathfrak{h})^{L}$, $\mathcal{R}(\mathfrak{h})$, respectively, and suppose that $c \leq 2$. Then $c^{\prime}=c$.

Proof. Clearly, $c \geq c^{\prime}$. We bring the situation $c=2, c^{\prime}=1$ to a contradiction (the other cases are obvious). If $c^{\prime}=1$, then the subalgebra $\mathfrak{z}_{\mathfrak{g}}(\mathfrak{h})$ is isomorphic to either $\mathfrak{s l}_{2}$ or $\mathrm{T}_{1}$. But an algebra of dimension 3 or 1 cannot have rank 2 .

\section{Classification of Simple embeddings}

From this moment on we assume that $\mathfrak{g}$ is a simple exceptional Lie algebra and $\mathfrak{h}$ is a semisimple subalgebra of $\mathfrak{g}$. We shall obtain a classification of the simple subalgebras up to conjugacy and then investigate the realizability in $\mathfrak{g}$ of their outer automorphisms. We shall thus arrive at a classification of the simple embeddings.

2.1. Identification of simple subalgebras. In [5] the simple subalgebras of exceptional Lie algebras are identified by their indices. In this subsection we explain these notions.

Let $\varphi: \mathfrak{h} \subset \mathfrak{g}$ be an embedding of a simple Lie algebra. We consider invariant scalar products $(,$,$) on the algebras \mathfrak{g}$ and $\mathfrak{h}$ normalized so that the greatest length of a root is equal to 2 . We define a new invariant scalar product $(x, y)_{1}=(\varphi(x), \varphi(y))$ on $\mathfrak{h}$. Then using [5], for some integer $j_{\varphi}$, called the index of the embedding $\varphi$, we have $(x, y)_{1}=j_{\varphi}(x, y)$.

Clearly, the index of a composite of embeddings is equal to the product of their indices. Furthermore, the index is invariant with respect to automorphisms of the algebra $\mathfrak{h}$. Therefore it makes sense to speak about the indices of simple subalgebras.

Suppose that embeddings $\varphi_{i}: \mathfrak{h} \hookrightarrow \mathfrak{g}, i=1, \ldots, k$, are such that their images commute. Then $j_{\varphi_{1}+\cdots+\varphi_{k}}=j_{\varphi_{1}}+\cdots+j_{\varphi_{k}}$.

As a rule, non-conjugate subalgebras have different indices. Therefore it is reasonable to denote simple subalgebras by indicating their index by a superscript, for example, $A_{1}^{28} \subset G_{2}$. If the indices are the same but the subalgebras are not linearly conjugate, then in addition we use primes: $A_{2}^{6^{\prime}}, A_{2}^{6^{\prime \prime}} \subset E_{8}$.

2.2. Dynkin's result. In this subsection we expound Dynkin's classification of the simple subalgebras of exceptional Lie algebras up to linear conjugacy. By Theorem 3 we are only interested in the non-regular subalgebras of rank greater than 1 of the algebras $\mathfrak{g}=E_{6}, E_{7}, E_{8}$. Note that we do not have to classify the S-subalgebras, since this has been done by Dynkin; we only need to find all their realizable automorphisms. 
In [5, Table 25] for each simple subalgebra $\mathfrak{h} \subset \mathfrak{g}$, up to linear conjugacy, the restrictions $\left.\pi_{1}\right|_{\mathfrak{h}}$ and ad $\left.\right|_{\mathfrak{h}}$ of the simplest and adjoint representations of the algebra $\mathfrak{g}$ to $\mathfrak{h}$ were indicated. (It is interesting to note that the representation ad $\left.\right|_{\mathfrak{h}}$ uniquely determines the linear conjugacy class of the subalgebra $\mathfrak{h}$.) Furthermore, the set $\mathcal{R}(\mathfrak{h})^{L S}$, known as the set of minimal ambient regular subalgebras, was indicated. This set consists of those regular subalgebras containing subalgebras $\mathfrak{h}^{\prime} \stackrel{L}{\sim} \mathfrak{h}$ in which $\mathfrak{h}^{\prime}$ is an S-subalgebra 6 We point out that the classification of the simple embeddings up to linear equivalence can easily be obtained from Dynkin's classification (Theorem 2).

In Dynkin's classification there is one inaccuracy, which is important for us: there does not exist a subalgebra $\mathfrak{s o}_{7} \subset E_{8}$ with unique minimal ambient regular subalgebra $2 D_{4}$. One can understand this by writing down the restrictions of the adjoint representation of the algebra $E_{8}$ to all possible subalgebras $\mathfrak{s o}_{7} \subset 2 D_{4}$ : it turns out that each of them is linearly conjugate to one of the subalgebras $\mathfrak{s o}_{7} \subset A_{6}, A_{7}^{\prime}$ or $\mathfrak{s o}_{7} \subset A_{7}^{\prime \prime}$. Furthermore, the subalgebras $\mathfrak{s l}_{3}$ and $\mathfrak{s l}_{5}$ of the algebra $E_{8}$ with unique minimal ambient regular subalgebras $E_{6}+A_{2}$ and $2 A_{4}$, respectively, are missing in [5, Table 25] (the fact that all the S-subalgebras $\mathfrak{s l}_{3} \subset E_{6}+A_{2}$ and $\mathfrak{s l}_{5} \subset 2 A_{4}$ are linearly conjugate can be verified directly by using Theorem 21).

Dynkin's result (more precisely, that part of it which we shall need) is presented in the first three columns of Tables 6, 7,8 ,

2.3. Description of Tables 6-8. The tables contain the classification up to equivalence of the simple non-regular embeddings of rank greater than 1 . We will obtain these results in this section. The first three columns of the tables contain the analogous classification up to linear equivalence (Dynkin's result).

In the first column we indicate, up to linear conjugacy, the subalgebras $\mathfrak{h} \subset \mathfrak{g}$ whose minimal ambient regular subalgebras are listed in the second column of the tables. We set $V$ to be the simplest module of the algebra $\mathfrak{g}$. We denote by $\Gamma(\mathfrak{h})^{L}$ the group of outer automorphisms of the subalgebra $R\left(\pi_{1}\right)(\mathfrak{h})$ that are realizable in $\mathfrak{g l}(V)$; in other words, the symmetry group of the diagram of the representation $\left.R\left(\pi_{1}\right)\right|_{\mathfrak{h}}$. The group $\Gamma(\mathfrak{h})^{L}$ enables one to obtain a classification of the simple embeddings up to linear equivalence. These groups, for the algebras $\mathfrak{h}$ that have outer automorphisms, are indicated in the third column of the tables.

The fourth column gives $n(\mathfrak{h})$, the number of conjugacy classes into which the linear conjugacy class of the subalgebra $\mathfrak{h}$ is partitioned. If the algebra $\mathfrak{h}$ has outer automorphisms, then the fifth column shows $\Gamma(\mathfrak{h})$, the group of the outer automorphisms of the subalgebra $\mathfrak{h}$ that are realizable in $\mathfrak{g}$, that is, the image of the natural homomorphism

$$
N_{G}(\mathfrak{h}) \rightarrow \text { Aut } \mathfrak{h} \rightarrow \text { Aut } \mathfrak{h} / \text { Int } \mathfrak{h} .
$$

Obviously, $\Gamma(\mathfrak{h}) \subset \Gamma(\mathfrak{h})^{L}$. In the cases where $n(\mathfrak{h})>1$, the groups $\Gamma(\mathfrak{h})$ for representatives of different conjugacy classes are listed separated by commas. The specifics of partitions of the corresponding linear equivalence classes is described by Theorem 7 .

2.4. Some remarks. In the rest of this section we will substantiate the results presented in the fourth and fifth columns of these tables. Our work is based on the information in the first three columns of Tables 6 8 and certain additional considerations. Table [5] (see [10]) is a great help in this; in it we interpret the symbol $\otimes$ as the tensor product of representations of simple ideals of the subalgebra $\mathfrak{r} \subset \mathfrak{g}$. For example, it follows from this table that all outer automorphisms of the subalgebra $D_{4}$ are realized in $F_{4}$

\footnotetext{
${ }^{6}$ The term "minimal ambient regular subalgebra" should not be understood literally: for example, in the case $\mathfrak{g}=F_{4}$ it could be the subalgebra $\mathfrak{r}=A_{1}+A_{1}$ : the diagonally embedded $\mathfrak{s l}_{2}$-subalgebra $\mathfrak{h} \subset \mathfrak{r}$ is regular in $\mathfrak{g}$ (its simple root corresponds to a short root of the algebra $\mathfrak{g}$ ), but is an S-subalgebra in $\mathfrak{r}$. However, in the cases $\mathfrak{g}=A_{n}, D_{n}, E_{6}, E_{7}, E_{8}$ there can be no such "surprises" [5, Theorem 2.4].
} 
(and therefore also in $E_{6}, E_{7}, E_{8}$ ). Consequently, all the S-subalgebras $\mathfrak{s o}_{7} \subset D_{4}$ are conjugate in $F_{4}$ (and in $E_{6}, E_{7}, E_{8}$ ). Indeed, up to conjugacy there are three such S-subalgebras defined by the following representations of the algebra $\mathfrak{s o}_{7}: R\left(\pi_{1}\right)+N, R\left(\pi_{3}\right)$, and $R\left(\pi_{3}\right)$ - the last representation defines an embedding that differs from the second by an outer automorphism of the algebra $\mathfrak{s o}_{8}$ (Theorem 4). These subalgebras are taken to one another by outer automorphisms of the algebra $\mathfrak{s o}_{8}$. This can easily be verified directly, but one can use the results of [6]: there does not exist an outer automorphism of order three of the algebra $\mathfrak{s o}_{8}$ that centralizes a subalgebra isomorphic to $\mathfrak{s o}_{7}$.

2.5. The case $\mathfrak{g}=\boldsymbol{E}_{\mathbf{6}}$. In this subsection we work with Table 6 First we prove that the contents of the fourth column are valid.

Classification of subalgebras. The subalgebras numbered 1-4 are S-subalgebras and their classification is known. We only observe that in cases 1 and 2 the subalgebras $\mathfrak{h}$ are not conjugate to their images under an outer automorphism of the algebra $\mathfrak{g}$ (see [5]). For the subalgebras numbered 5-13 the results are obvious (they follow from the notion of Ssubalgebra). Case 14 has already been examined. We consider separately the subalgebras numbered 15, 16, 17.

Case 15. In the algebra $\mathfrak{s o}_{10}$ up to conjugacy there are two S-subalgebras $\mathfrak{s o}_{5}$ embedded via the adjoint representation (see Example 1). We denote them by $\mathfrak{h}_{1}$ and $\mathfrak{h}_{2}$. It follows from Table 6 that they are linearly conjugate in $\mathfrak{g}$. If they were conjugate in $\mathfrak{g}$, then by Proposition 3 they would be conjugate by an element normalizing $D_{5}$. But it is clear from Table 5 that no outer automorphism of the subalgebra $D_{5}$ is realized in $\mathfrak{g}$. Consequently, the subalgebras $\mathfrak{h}_{1}$ and $\mathfrak{h}_{2}$ are not conjugate.

Cases 16, 17. We set $\mathfrak{r}=3 A_{2}$ and analyze the S-subalgebras $\mathfrak{h} \subset \mathfrak{r}$ isomorphic to $\mathfrak{s l}_{3}$. We enumerate them up to conjugacy in $\mathfrak{r}$ :

$$
\begin{aligned}
& \mathfrak{h}_{1}=\left\{x+x+x: x \in \mathfrak{s l}_{3}\right\}, \\
& \mathfrak{h}_{2}=\left\{x+\sigma(x)+x: x \in \mathfrak{s l}_{3}\right\}, \\
& \mathfrak{h}_{3}=\left\{x+x+\sigma(x): x \in \mathfrak{s l}_{3}\right\}, \\
& \mathfrak{h}_{4}=\left\{\sigma(x)+x+x: x \in \mathfrak{s l}_{3}\right\},
\end{aligned}
$$

where $\sigma$ is an outer automorphism of the algebra $\mathfrak{s l}_{3}$. It is clear from Table 5 that the transpositions of the first two (respectively, last two) simple ideals of the subalgebra $\mathfrak{r}$ simultaneously with an outer automorphism of the third (respectively, the first) ideal are realized in $\mathfrak{g}$. Consequently, the subalgebras $\mathfrak{h}_{1}, \mathfrak{h}_{3}$, and $\mathfrak{h}_{4}$ are conjugate in $\mathfrak{g}$. Therefore there are at most two conjugacy classes corresponding to the subalgebras listed above. On the other hand, according to Dynkin's result there are exactly two of them, since there exist two linear conjugacy classes of the subalgebras under consideration. For definiteness we note that case 16 corresponds to the subalgebra $\mathfrak{h}_{2}$, and case 17 to the subalgebra $\mathfrak{h}_{1}$. It remains to prove that the S-subalgebras $\mathfrak{s l}_{3} \subset D_{4}$ and $\mathfrak{h}_{2} \subset \mathfrak{r}$ are conjugate. But this follows from Proposition 7 and the fact that both S-subalgebras $\mathfrak{s l}_{3} \subset D_{4}$ (they are embedded via the adjoint representation) are conjugate in $\mathfrak{g}$. We note also here that the outer automorphisms of the algebras $\mathfrak{h}_{1}, \mathfrak{h}_{2}$ are realized by elements of $N_{G}(\mathfrak{r})$.

We now pass to the classification of embeddings, that is, to verifying the last column of Table 6 .

Classification of embeddings. In all cases apart from the subalgebras with number 1 , it is easy to verify that $\Gamma(\mathfrak{h})^{L}=\Gamma(\mathfrak{h})$ and all automorphisms are realized by elements of $N_{G}(\mathfrak{r})$ for some minimal ambient regular subalgebra $\mathfrak{r} \supset \mathfrak{h}$. We now examine case 1 . It is clearly sufficient to find the group $\Gamma(\mathfrak{h})$ only for a representative of one of the conjugacy 
classes (recall that the latter are taken to one another by an outer automorphism of the algebra $\mathfrak{g})$.

We set $\left\{e_{i}, h_{i}, f_{i}\right\}, i=1, \ldots, 6$, to be the standard generators of the three-dimensional subalgebras corresponding to the simple roots of the algebra $\mathfrak{g}$ and set $e_{i j k}=\left[\left[e_{i}, e_{j}\right], e_{k}\right]$, $i, j, k=1, \ldots, 6$. Then the following vectors are the root vectors corresponding to the simple roots $x$ and $y$ of the S-subalgebra $\mathfrak{h}=\mathfrak{s l}_{3} \subset \mathfrak{g} \cdot 7$

$$
\begin{aligned}
& e_{x}=\sqrt{2}\left(e_{1}+e_{2}+e_{4}+e_{5}\right)+e_{6}, \\
& f_{x}=\sqrt{2}\left(f_{1}+f_{2}+f_{4}+f_{5}\right)+f_{6}, \\
& e_{y}=(-1+i) e_{123}+\sqrt{2}\left(e_{634}-e_{236}\right)+(1+i) e_{345}+i e_{234}, \\
& f_{y}=(-1-i) f_{123}+\sqrt{2}\left(f_{634}-f_{236}\right)+(1-i) f_{345}-i f_{234} .
\end{aligned}
$$

We consider the involution $\theta$ of the algebra $\mathfrak{g}$ defined by the longest element of its Weyl group: $\theta\left(e_{i}\right)=-f_{6-i}, i=1, \ldots, 5$, and $\theta\left(e_{6}\right)=-f_{6}$. Then it is easy to see that $\theta(\mathfrak{h})=\mathfrak{h}, \theta\left(e_{x}\right)=-f_{x}$ and $\theta\left(e_{y}\right)=f_{y}$. Therefore the automorphism $\theta$ induces an outer automorphism of the subalgebra $\mathfrak{h}$ and $\Gamma(\mathfrak{h})=\mathbb{Z}_{2}$.

This completes the analysis of the case $\mathfrak{g}=E_{6}$.

\subsection{The case $\mathfrak{g}=\boldsymbol{E}_{\mathbf{7}}$.}

Classification of subalgebras. Subalgebra number 1 in Table 7 is an S-subalgebra, and there is nothing to prove here. Subalgebras numbered 2-18 are linearly conjugate to subalgebras of $E_{6}$. Cases 2-12 have already been examined in the preceding subsection. Since an outer automorphism of the subalgebra $E_{6}$ is realized in $\mathfrak{g}$ (Proposition 2), we conclude that in cases 13-15 the linear conjugacy classes are not partitioned. In cases 16, 17 (as well as in cases 24, 25) the fourth column is substantiated by using Proposition 7 . The linearly conjugate subalgebras numbered 18 are conjugate. This follows from Proposition [6] it is sufficient to prove that $\operatorname{dim} \mathfrak{z} \mathfrak{g}(\mathfrak{h})>3$. But this is obvious, since $\mathfrak{z} \mathfrak{g}\left(A_{4}\right) \supset A_{2}$. Cases 19-23 obviously follow from the classification of the S-subalgebras of classical Lie algebras.

Cases 26, 27. In the algebra $\mathfrak{s l}_{6}+\mathfrak{s l}_{3}$, there are two (up to conjugacy) S-subalgebras isomorphic to $\mathfrak{s l}_{3}$ (the latter embeds into $\mathfrak{s l}_{6}$ via the symmetric square of its tautological representation). It is clear from Table 7 that these subalgebras are not linearly conjugate. Therefore we only need to prove that there exist two regular subalgebras

$$
\mathfrak{r}_{1}=A_{7}, \quad \mathfrak{r}_{2}=A_{5}+A_{2} \subset \mathfrak{g}
$$

that intersect in a subalgebra which is isomorphic to $\mathfrak{s l}_{3}$ and is an S-subalgebra in each of them. In any case, they intersect in the principal three-dimensional subalgebra $\mathfrak{s} \subset \mathfrak{s l}_{3}$ (Theorem 3). We know from [1] that $Z_{G}(\mathfrak{s}) \simeq \mathrm{SO}_{3}$. We observe that $Z_{G}\left(\mathfrak{r}_{1}\right) \simeq \mathbb{Z}_{2}$ and $Z_{G}\left(\mathfrak{r}_{2}\right) \simeq \mathbb{Z}_{3}$ (see [6]). It is known that any two isomorphic finite subgroups of $\mathrm{SO}_{3}$ are conjugate. Therefore we are not interested in the nature of the embedding of any particular subgroup into $\mathrm{SO}_{3}$.

We set $Z=\mathbb{A}_{4} \subset \mathrm{SO}_{3}$ and claim that $\mathfrak{z}_{\mathfrak{g}}(Z) \simeq \mathfrak{s l}_{3}$. Since the subalgebra $\mathfrak{s o}_{8} \subset \mathfrak{s l}_{8}$ is the centralizer of some involution of the algebra $\mathfrak{s l}_{8}$, we conclude that $Z_{G}\left(\mathfrak{s o}_{8}\right)=\mathbb{V}_{4} \subset Z$ (this will be proved more formally in Example 11). We have the factorization

$$
\mathbb{A}_{4}=\mathbb{Z}_{3}<\mathbb{V}_{4}
$$

\footnotetext{
${ }^{7}$ There is a misprint in [5] Table 24]: the coefficient of $f_{234}$ in the formula for $f_{y}$ is equal to $-i$, not to $i$. This follows from the preceding relations given there, or can be verified by a straightforward calculation using the formulae given here.
} 
Therefore on the subalgebra $\mathfrak{s o}_{8} \subset \mathfrak{g}$ there arises a (non-trivial) grading such that the elements of the zero component form the subalgebra $\mathfrak{s o}_{8}^{0}=\mathfrak{z}_{\mathfrak{g}}(Z)$. This grading cannot be an inner one, since one can verify that $\mathfrak{s} \subset \mathfrak{s o}_{8}$ is an S-subalgebra. Therefore, by [ 6 , the algebra $\mathfrak{s o}_{8}^{0}$ is isomorphic to either $\mathfrak{s l}_{3}$ or $G_{2}$. The last case is impossible: $G_{2} \not \subset$ $\mathfrak{z}_{\mathfrak{g}}\left(\mathbb{Z}_{3}\right)=\mathfrak{r}_{2}$. Therefore $\mathfrak{z}_{\mathfrak{g}}(Z)=\mathfrak{s l}_{3}$ is an S-subalgebra in $\mathfrak{s o}_{8}$ and, consequently, also in $\mathfrak{r}_{1}$. Thus, the analysis of this case is complete.

Classification of embeddings. All the cases, except for 1 and 22, can be trivially examined by using Proposition 2. We now find the groups $\Gamma(\mathfrak{h})$ for the subalgebras with the aforementioned numbers.

Case 1. This case is examined similarly to case 1 where $\mathfrak{g}=E_{6}$. Using the same notation we give the formulae for the root vectors of the S-subalgebra $\mathfrak{h}=\mathfrak{s l}_{3} \subset \mathfrak{g} 8$

$$
\begin{aligned}
& e_{x}=e_{2}+\sqrt{6}\left(e_{4}+e_{5}\right)+2\left(e_{6}+e_{7}\right), \\
& f_{x}=f_{2}+\sqrt{6}\left(f_{4}+f_{5}\right)+2\left(f_{6}+f_{7}\right), \\
& e_{y}=e_{4357}+\sqrt{6}\left(e_{1}-e_{2347}\right)+2\left(e_{2345}+e_{3456}\right), \\
& f_{y}=f_{4357}+\sqrt{6}\left(f_{1}-f_{2347}\right)+2\left(f_{2345}+f_{3456}\right) .
\end{aligned}
$$

It is obvious that the automorphism of the algebra $\mathfrak{g}$ corresponding to the longest element of its Weyl group realizes an outer automorphism of the subalgebra $\mathfrak{h}$.

Case 22. Let $\mathfrak{s}$ be the principal three-dimensional subalgebra of the algebra $\mathfrak{h}=\mathfrak{s o}_{8}$. Then $Z_{G}(\mathfrak{s}) \simeq \mathrm{SO}_{3}$, 1]. We already know that $Z_{G}(\mathfrak{h})=\mathbb{V}_{4} \subset \mathrm{SO}_{3}$. Since the group $\mathrm{SO}_{3}$ has a subgroup isomorphic to $\mathbb{S}_{4}$, we obtain that there exists a subgroup $\mathbb{S}_{3} \subset \mathrm{SO}_{3}$ acting on the subalgebra $\mathfrak{h}$. It is clear that this subgroup acts by outer automorphisms. Thus the result presented in the fifth column of Table 7 is completely substantiated.

2.7. The case $\mathfrak{g}=\boldsymbol{E}_{\mathbf{8}}$. We now prove the results presented in the fourth and fifth columns of Table 8 .

Classification of subalgebras. The subalgebra with number 1 is an S-subalgebra and is of no interest. Corresponding to the numbers 2-17 (respectively, 18-24) are the subalgebras linearly conjugate to subalgebras in $E_{6}$ (respectively, $E_{7}$ ). We can examine cases 2-13 (respectively, 18-20) in the same fashion as the case $\mathfrak{g}=E_{6}$ (respectively, $\mathfrak{g}=E_{7}$ ). Cases 14-16 have already been analyzed in the preceding subsection.

To prove that the linear conjugacy class in case 17 is not partitioned it is sufficient to prove that an outer automorphism of the subalgebra $A_{2}$ is realized in $\mathfrak{z}_{\mathfrak{g}}\left(A_{2}\right)=E_{6}$. But this is obvious, since it is realized even in $G_{2} \subset E_{6}$.

Cases 21, 22, 36 are analyzed by using Proposition 7 and in cases 27-35 the substantiation of the fourth column is based on the classification of the S-subalgebras and offers no difficulty.

We now prove that the linearly conjugate subalgebras corresponding to number 37 (or 38) are conjugate. For that it is sufficient to prove that the outer automorphisms of the subalgebra $D_{4}$ (respectively, $A_{3}$ ) are realized in $\mathfrak{z}_{\mathfrak{g}}\left(A_{2}\right)=E_{6}$ (respectively, $\mathfrak{z}_{\mathfrak{g}}\left(A_{3}\right)=$ $\left.D_{5}\right)$. But we already know that, up to conjugacy, there are two subalgebras $A_{3} \subset D_{5}$ corresponding to the representations $R\left(\pi_{1}\right)+R\left(\pi_{3}\right)+2 N$ and $R\left(\pi_{2}\right)+4 N$; in either case an outer automorphism is realized.

Cases 39, 40 can easily be examined using Proposition 5.

It remains to consider the subalgebras numbered $23-26$ and $41-43$.

${ }^{8} \mathrm{~A}$ misprint again slipped into [5] Table 24]: the scalar product $\left(\alpha_{7}^{\vee}, h_{y}\right)$ is equal to -1 , not to 1 ; this gives rise to an error in the formulae for $e_{y}, f_{y}$. 
Cases 24-26. Using Proposition 7 and the fact that all the subalgebras $\mathfrak{s o}_{8} \subset \mathfrak{s l}_{8}$ are conjugate we arrive at the conclusion that, in the cases under consideration, any of the subalgebras $\mathfrak{h} \subset \mathfrak{g}$ embeds into $\mathfrak{r}=2 D_{4}$. For convenience we give the Dynkin diagram $D_{4}$ (Figure 1).

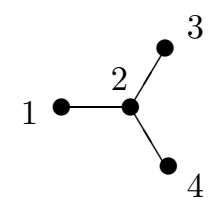

Figure 1. Dynkin diagram $D_{4}$

We denote the symmetries of the diagram with fixed vertices $i, 2$ by $\sigma_{i}, i=1,3,4$, and the cycle (134) by $\theta$. We denote the corresponding automorphisms of the algebra by the same letters. In the algebra $\mathfrak{r}$ there are 6 conjugacy classes of S-subalgebras isomorphic to $\mathfrak{s o}_{8}$ with representatives

$$
\begin{aligned}
\mathfrak{h}_{2} & =\left\{x+x: x \in \mathfrak{s o}_{8}\right\}, \\
\mathfrak{h}_{i} & =\left\{x+\sigma_{i}(x): x \in \mathfrak{s o}_{8}\right\}, \quad i=1,3,4, \\
\mathfrak{h}_{5} & =\left\{x+\theta(x): x \in \mathfrak{s o}_{8}\right\}, \\
\mathfrak{h}_{6} & =\left\{x+\theta^{-1}(x): x \in \mathfrak{s o}_{8}\right\} .
\end{aligned}
$$

Using Table 5 we find that simultaneous outer automorphisms of the simple ideals of the subalgebra $\mathfrak{r}$, as well as their permutation, are realized in $\mathfrak{g}$. Therefore the subalgebras $\mathfrak{h}_{i}, i=1,3,4$, are conjugate. The same can also be said about the subalgebras $\mathfrak{h}_{5}, \mathfrak{h}_{6}$. Consequently, we have at most three conjugacy classes of S-subalgebras $\mathfrak{s o}_{8} \subset \mathfrak{r}$. On the other hand, it is clear from Table 8 that there exist exactly three linear conjugacy classes of such subalgebras. Therefore they coincide with the conjugacy classes. Writing down the restrictions of the adjoint representation of the algebra $\mathfrak{g}$ to the subalgebras $\mathfrak{h}_{i}, i=1, \ldots, 6$, one can verify that the subalgebras $\mathfrak{h}_{1}, \mathfrak{h}_{2}$ are linearly conjugate (and therefore conjugate) to subalgebras in $A_{7}^{\prime}, A_{7}^{\prime \prime}$, respectively.

It is easy to see that $\Gamma\left(\mathfrak{h}_{i}\right)^{L}=\Gamma\left(\mathfrak{h}_{i}\right), i=1, \ldots, 6$, and the corresponding outer automorphisms are realized by elements of $N_{G}(\mathfrak{r})$. It is interesting to note that this fact implies (by Proposition 3, $\widetilde{\mathfrak{r}}=E_{7}$ ) that all the outer automorphisms of subalgebra with number 22 in Table 7 are realizable in $\mathfrak{g}=E_{7}$.

Case 23. Using Proposition 7 we obtain that every subalgebra that is linearly conjugate to $\mathfrak{h}$ is conjugate to one of the S-subalgebras $\mathfrak{h}_{1} \subset A_{7}^{\prime}$ or $\mathfrak{h}_{2} \subset A_{7}^{\prime \prime}$. We know from [5] that the subalgebra $\mathfrak{n}_{\mathfrak{g}}\left(\mathfrak{h}_{1}\right) \simeq \mathfrak{s l}_{3}+\mathfrak{s l}_{2}$ is a (maximal) S-subalgebra in $\mathfrak{g}$; this cannot be said about $\mathfrak{n}_{\mathfrak{g}}\left(\mathfrak{h}_{2}\right)=\mathfrak{h}_{2}+A_{1}$, which is obviously an R-subalgebra. Therefore the subalgebras $\mathfrak{h}_{1}$ and $\mathfrak{h}_{2}$ are not conjugate (and, moreover, their centralizers are not conjugate, although they are isomorphic).

Note that the subalgebras $\mathfrak{h}_{1}$ and $\mathfrak{h}_{2}$ embed into the subalgebras with numbers 26 and 24 , respectively. In particular, they are contained in $2 D_{4}$.

Case 41. In the notation of the analysis of cases 26,27 for $\mathfrak{g}=E_{7}$ we have

$$
\mathfrak{r}_{1}=2 A_{4}, \quad \mathfrak{r}_{2}=A_{3}+D_{5}, \quad Z_{G}(\mathfrak{s}) \simeq \mathbb{S}_{5}, \quad Z_{G}\left(\mathfrak{r}_{1}\right)=\mathbb{Z}_{5} \subset \mathbb{S}_{5}, \quad Z_{G}\left(\mathfrak{r}_{2}\right)=\mathbb{Z}_{4} \subset \mathbb{S}_{5} .
$$

Since all the S-subalgebras $\mathfrak{s o}_{5} \subset \mathfrak{r}_{1}, \mathfrak{s o}_{5} \subset \mathfrak{r}_{2}$ in each case are conjugate (in the last case this follows from Proposition 21), it is sufficient to prove that there exist subalgebras that are conjugate to $\mathfrak{r}_{1}, \mathfrak{r}_{2}$ and contain a common S-subalgebra isomorphic to $\mathfrak{s o}_{5}$. 
We observe that in $\mathbb{S}_{5}$ there exists a subgroup $\mathbb{Z}_{4}<\mathbb{Z}_{5}$ (the factors are generated by the cycles (2354) and (12345), respectively). This means that in the algebra $\mathfrak{g}$ there exist subalgebras conjugate to $\mathfrak{r}_{1}, \mathfrak{r}_{2}$ (which we denote by the same symbols $\mathfrak{r}_{1}, \mathfrak{r}_{2}$ ) that intersect in the subalgebra $\mathfrak{r}_{1}^{0}$ consisting of the elements of zero degree with respect to some $\mathbb{Z}_{4}$-grading on $\mathfrak{r}_{1}$. This grading is not an inner one, since $\mathfrak{s} \subset \mathfrak{r}_{1}$ is an S-subalgebra. Then it is clear that $\mathfrak{r}_{1}^{0}$ is the required subalgebra $\mathfrak{s o}_{5}$.

Cases 42, 43. In the algebra $\mathfrak{s o}_{16}$ there are exactly two non-conjugate subalgebras isomorphic to $\mathfrak{s o}_{9}$, which embed into it via the spinor representation and are taken to one another by an outer automorphism of the algebra $\mathfrak{s o}_{16}$. It is clear from Table 8 that these subalgebras are not linearly conjugate in $\mathfrak{g}=E_{8}$. Therefore it is sufficient to consider only case 42 .

In the notation of the analysis of the preceding case we have

$$
\mathfrak{r}_{1}=A_{8}, \quad \mathfrak{r}_{2}=D_{8}, \quad Z_{G}(\mathfrak{s}) \simeq \mathbb{S}_{3}, \quad Z_{G}\left(\mathfrak{r}_{1}\right)=\mathbb{Z}_{3} \subset \mathbb{S}_{3}, \quad Z_{G}\left(\mathfrak{r}_{2}\right)=\mathbb{Z}_{2} \subset \mathbb{S}_{3} .
$$

Since $\mathbb{S}_{3}=\mathbb{Z}_{2} \wedge \mathbb{Z}_{3}$, it obviously follows that the linear conjugacy class of the subalgebra $\mathfrak{h} \subset \mathfrak{g}$ is not partitioned also in this case: $\mathfrak{r}_{1}^{0} \simeq \mathfrak{s o}_{9}$.

Classification of embeddings. All the cases that we have not considered here can easily be examined using Propositions 2, 5.

This completes the classification of the simple embeddings.

\section{InVARIANts of exceptional Lie algebras}

Let $H \subset G$ be a reductive subgroup of a reductive Lie group, and let $\mathfrak{h} \subset \mathfrak{g}$ be the corresponding inclusion of Lie algebras. We consider the adjoint action Ad of the group $G$. The corresponding algebra of invariant polynomials is denoted by $\mathbb{C}[\mathfrak{g}]^{G}$. The algebra of restrictions of these functions to the subspace $\mathfrak{h}$ is denoted by $\mathbb{C}[\mathfrak{h}]^{G}$. Its spectrum $\mathfrak{h} / / G$ coincides with the closure of the set $\pi_{G}(\mathfrak{h})$ in $\mathfrak{g} / / G$, where $\pi_{G}: \mathfrak{g} \rightarrow \mathfrak{g} / / G$ is the factorization morphism. Let $\psi: \mathfrak{h} / / H \rightarrow \mathfrak{h} / / G$ be the morphism corresponding to the inclusion $\mathbb{C}[\mathfrak{h}]^{G} \subset \mathbb{C}[\mathfrak{h}]^{H}$. By $[8$, we know that the morphism $\psi$ is finite.

We consider the chain of inclusions

$$
G_{2} \subset D_{4} \subset F_{4} \subset E_{6} \subset E_{7} .
$$

The next theorem was proved in 8$]$.

Theorem 5. The following conditions are equivalent:

1. For any two embeddings of the torus $\varphi_{i}: \mathfrak{t} \subset \mathfrak{h}, i=1,2$, such that $\varphi_{2}=\operatorname{Ad} g \circ \varphi_{1}$, $g \in G$, it follows that $\varphi_{2}=\operatorname{Ad} h \circ \varphi_{1}, h \in H$.

2. For any semisimple element $h \in H$ we have $G h \cap H=H h$.

3. The map $\psi$ is bijective.

By (2)-(5) the conditions of Theorem 5 are satisfied for the pairs $H=G_{2}, G=F_{4}$ and $H=F_{4}, G=E_{6}$. In this section we show that these conditions hold in the case of any inclusion $\mathfrak{h} \subset \mathfrak{g}$ in (6). Here the roles of the groups are played by $G=\operatorname{Int} \mathfrak{g}$, $H=N_{G}(\mathfrak{h}) \simeq$ Aut $\mathfrak{h}$. The absence of the algebra $E_{8}$ in (6) is explained by the fact that the conditions of Theorem 5 are not satisfied for the inclusion $E_{7} \subset E_{8}$ : the regular subalgebras $A_{5}^{\prime}$ and $A_{5}^{\prime \prime}$ in $E_{7}$ obtained from one another by an outer automorphism of $D_{6} \subset E_{7}$ are not linearly conjugate in $E_{7}$, but are linearly conjugate in $E_{8}$ (since $\left.D_{6} \subset B_{6} \subset E_{8}\right)$.

Theorem 6. If $\mathfrak{h} \subset \mathfrak{g}$ is an inclusion of (not necessarily consecutive) subalgebras in (6), then

$$
\mathbb{C}[\mathfrak{h}]^{G}=\mathbb{C}[\mathfrak{h}]^{H} .
$$


We reduce the proof to the case $\mathfrak{h}=E_{6}, \mathfrak{g}=E_{7}$. First we observe that it is sufficient to prove Theorem 6 for consecutive inclusions.

Lemma 1. Let $\mathfrak{h}_{1} \subset \mathfrak{h}_{2} \subset \cdots \subset \mathfrak{h}_{n}$ be inclusions of semisimple Lie algebras. Suppose that for any pair of consecutive inclusions $\mathfrak{h}_{i} \subset \mathfrak{h}_{i+1}, i=1, \ldots, n-2$, there exist outer automorphisms of the algebra $\mathfrak{h}_{i+1}$ centralizing $\mathfrak{h}_{i}$ and generating the group of outer automorphisms Aut $\mathfrak{h}_{i+1} / \operatorname{Int} \mathfrak{h}_{i+1}$ of the algebra $\mathfrak{h}_{i+1}$. Then if $\mathbb{C}\left[\mathfrak{h}_{i}\right]^{H_{k}}=\mathbb{C}\left[\mathfrak{h}_{i}\right]^{N_{H_{k}}\left(\mathfrak{h}_{i}\right)}$ holds for $i=k-1=1, \ldots, n-1$, where $H_{i}=\operatorname{Int} \mathfrak{h}_{i}$, it holds for any $i<k, i, k=1, \ldots, n$.

Proof. It is sufficient to prove the lemma in the case $i=1, k=n$. Using induction on $k$ we can assume that the assertion holds for $k=n-1$. We now prove it in the case $k=n \geq 3$. Indeed, we have the chain of equalities

$$
\begin{aligned}
\mathbb{C}\left[\mathfrak{h}_{1}\right]^{H_{n}} & =\left.\mathbb{C}\left[\mathfrak{h}_{n}\right]^{H_{n}}\right|_{\mathfrak{h}_{1}}=\left.\left(\left.\mathbb{C}\left[\mathfrak{h}_{n}\right]^{H_{n}}\right|_{\mathfrak{h}_{n-1}}\right)\right|_{\mathfrak{h}_{1}}=\left.\mathbb{C}\left[\mathfrak{h}_{n-1}\right]^{H_{n}}\right|_{\mathfrak{h}_{1}}=\left.\left(\mathbb{C}\left[\mathfrak{h}_{n-1}\right]^{N_{H_{n}}\left(\mathfrak{h}_{n-1}\right)}\right)\right|_{\mathfrak{h}_{1}} \\
& =\left.\left(\mathbb{C}\left[\mathfrak{h}_{n-1}\right]^{H_{n-1}}\right)\right|_{\mathfrak{h}_{1}}=\mathbb{C}\left[\mathfrak{h}_{1}\right]^{H_{n-1}}=\mathbb{C}\left[\mathfrak{h}_{1}\right]^{N_{H_{n-1}}\left(\mathfrak{h}_{1}\right)} \supset \mathbb{C}\left[\mathfrak{h}_{1}\right]^{N_{H_{n}}\left(\mathfrak{h}_{1}\right)} .
\end{aligned}
$$

Therefore, $\mathbb{C}\left[\mathfrak{h}_{1}\right]^{H_{n}}=\mathbb{C}\left[\mathfrak{h}_{1}\right]^{N_{H_{n}}\left(\mathfrak{h}_{1}\right)}$, as required.

Obviously, Lemma 1 will be applicable to the chain (6) if we prove Theorem 6 for consecutive inclusions in (6). To do this, we reproduce Table 1 listing the degrees of the generators for the algebra $\mathbb{C}[\mathfrak{g}]^{G}$ (see, for example, [3, Reference Chapter]).

TABle 1. Degrees of the generators of the algebra $\mathbb{C}[\mathfrak{g}]^{G}$

\begin{tabular}{|c|c|}
\hline $\mathfrak{g}$ & $\mathbb{C}[\mathfrak{g}]^{G}$ \\
\hline$G_{2}$ & 2,6 \\
\hline$D_{4}$ & $2,4,4,6$ \\
\hline$F_{4}$ & $2,6,8,12$ \\
\hline$E_{6}$ & $2,5,6,8,9,12$ \\
\hline$E_{7}$ & $2,6,8,10,12,14,18$ \\
\hline
\end{tabular}

It is known that if $\mathfrak{g}$ is a simple Lie algebra, then the algebra $\mathbb{C}[\mathfrak{g}]^{\operatorname{Int} \mathfrak{g}}$ is generated by the functions $\operatorname{tr} \pi_{1}\left(x^{k}\right)$, where $x \in \mathfrak{g}$ and $k$ runs over the set of degrees of a minimal system of generators of the algebra (see, for example, 44). This fact and Table 1 imply that Theorem 6 holds for the inclusion $G_{2} \subset D_{4}$ (since $\left.\pi_{D_{4}}\right|_{G_{2}} \sim \pi_{G_{2}}+N$ ). It follows from relation (3) that this theorem also holds in the case $F_{4} \subset E_{6}$. In the case $D_{4} \subset F_{4}$ one must pass to the maximal tori (Chevalley's theorem) and observe that the Weyl group of $F_{4}$ is obtained as an extension of the Weyl group of $D_{4}$ by the group $\mathbb{S}_{3}$. Thus, it remains to prove the following.

Proposition 8. We have the equality $\mathbb{C}\left[E_{6}\right]^{E_{7}}=\mathbb{C}\left[E_{6}\right]^{\text {Aut } E_{6}}$.

In the proof we shall need the following lemma.

Lemma 2. Let $A \subset B$ be an extension of commutative graded finitely generated algebras. Suppose that in $A$ and $B$ one can choose homogeneous generators $f_{i} \in A, g_{i} \in B$, $i=1, \ldots, n$, so that the following conditions hold:

1. $\operatorname{deg} f_{i}=\operatorname{deg} g_{i}$;

2. the system of generators $f_{i} \in A$ is minimal.

Then $A=B$. 
Proof. We need to prove that $g_{i} \in A$ for all $i=1, \ldots, n$. We use induction on the degree. Let $g_{1}, \ldots, g_{k_{1}}$ be the generators of degree 1 (which may not exist). Then $f_{1}, \ldots, f_{k_{1}}$ can be expressed as linear combinations of the $g_{i}$. If the corresponding matrix is singular, then we obtain a contradiction to the fact that the system of generators of the algebra $A$ is minimal. Therefore, $g_{1}, \ldots, g_{k_{1}} \in A$. The induction step for degree $d>1$ can be performed in a similar fashion taking into account the fact that the elements of the algebra $B$ of degrees less than $d$ belong to the algebra $A$.

Proof of Proposition 8, We take

$$
A=\mathbb{C}\left[E_{6}\right]^{E_{7}}, \quad B=\mathbb{C}\left[E_{6}\right]^{\text {Aut } E_{6}}
$$

for the algebras $A$ and $B$ in the hypothesis of the lemma. In each of them we can choose generators of degrees $2,6,8,10,12,14,18$. For the algebra $A$ this follows from Table 1 . We now prove this for $B$. Let $C=\mathbb{C}\left[E_{6}\right]^{\text {Int } E_{6}}=\mathbb{C}\left[\chi_{2}, \chi_{5}, \chi_{6}, \chi_{8}, \chi_{9}, \chi_{12}\right]$, where $\chi_{k}$ is the invariant of degree $k$. Then the elements $\chi_{2}, \chi_{6}, \chi_{8}, \chi_{5}^{2}, \chi_{12}, \chi_{5} \chi_{9}, \chi_{9}^{2}$ belong to the algebra $B$. They actually generate it, since a Weyl involution of the algebra $E_{6}$ acts on the elements of $C$ of odd degree as multiplication by -1 and the algebra $C$ is free.

To apply the lemma we only need to verify that the chosen system of generators for $A$ satisfies the minimality condition. Since the morphism $\psi$ is finite, it follows that tr.deg Quot $A=6$. Therefore it is sufficient to prove that the algebra $A$ is not freely generated. It follows from the results of $[8]$ that the morphism $\psi$ is birational (consequently, it is a normalization) if the groups $G$ and $H$ are simple and all the outer automorphisms of the subgroup $H$ are realized in $G$. In particular, this holds in our case. If the algebra $A$ were freely generated, then the variety $\mathfrak{h} / / G$ would be isomorphic to a vector space and would coincide with its normalization, which is impossible, since the algebra $B$ is not free (the corresponding "Weyl group" is not generated by reflections).

It remains to use the lemma to obtain the required result.

\section{Classification of Semisimple Embeddings}

In this section we determine all the classes of linearly equivalent embeddings that are partitioned into several classes of equivalent embeddings. It remains to consider the embeddings of non-simple semisimple Lie algebras $\mathfrak{h}$ into the exceptional Lie algebras $\mathfrak{g}=E_{6}, E_{7}, E_{8}$.

4.1. Dynkin characteristics of three-dimensional subalgebras. This subsection is auxiliary. Here we expound some results from [5] that will be useful in this section.

The (Dynkin) characteristic of a simple three-dimensional subalgebra $\mathfrak{s}=\langle e, h, f\rangle \subset \mathfrak{g}$ is the element $h$ of a Cartan subalgebra. By Theorem 3 the subalgebra $\mathfrak{s}$ is determined uniquely by its characteristic, up to conjugacy. We can assume that the vector $h$ lies in some fixed Weyl chamber. Then this element is given by the set of non-negative integers (labels) $\alpha_{i}(h)$, where $\alpha_{i}, i=1, \ldots, n$, are the simple roots of the algebra $\mathfrak{g}$. One can prove that these labels are equal to 0,1 , or 2 . For example, on the characteristic of the principal three-dimensional subalgebra all the simple roots take value 2 .

Dynkin found the characteristics of all the simple three-dimensional subalgebras of exceptional Lie algebras [5, Tables 16-20]. From this, in turn, one can easily obtain a list of the subalgebras $\mathfrak{z}_{\mathfrak{g}}(h)$, where $h$ is the characteristic of some three-dimensional subalgebra. The system of simple roots of the derived subalgebra of such a subalgebra consists of those roots $\alpha_{i}$ that are equal to 0 on $h$. 
4.2. The main idea. Let $\mathfrak{h}=\mathfrak{h}_{1} \oplus \mathfrak{h}_{2}$ be a decomposition into a sum of non-zero ideals and let $\mathfrak{k} \subset \mathfrak{h}, \mathfrak{k}_{i} \subset \mathfrak{h}_{i}, i=1,2$, be Cartan subalgebras. In what follows we assume that we are given an embedding $\varphi: \mathfrak{h} \hookrightarrow \mathfrak{g}$. We are interested in how uniquely this embedding is determined by the embedding $\left.\varphi\right|_{\mathfrak{k}}$, namely, whether in the linear equivalence class of $\varphi$ there is a non-equivalent embedding. We call an embedding $\varphi$ whose linear equivalence class is partitioned, and the corresponding subalgebra $\varphi(\mathfrak{h}) \subset \mathfrak{g}$, interesting; otherwise they are uninteresting.

We regard the algebra $\mathfrak{h}$ as a subalgebra of $\mathfrak{g}$ bearing in mind the embedding $\varphi$. We set $\mathfrak{a}_{i}=\mathfrak{z}_{\mathfrak{g}}\left(\mathfrak{h}_{i}\right), \mathfrak{z}_{i}=\mathfrak{z}_{\mathfrak{g}}\left(\mathfrak{k}_{i}\right), i=1,2$. It is clear that $\mathfrak{a}_{i} \subset \mathfrak{z}_{i}$ and the derived subalgebra of $\mathfrak{z}_{i}$ is a complete regular subalgebra of $\mathfrak{g}$.

Suppose that the algebra $\mathfrak{z}_{1}$ contains no simple ideals of types $D_{n}(n \geq 4), E_{6}$ or $E_{7}$. Then by Theorem 3 the embedding $\left.\varphi\right|_{\mathfrak{h}_{2}}: \mathfrak{h}_{2} \hookrightarrow \mathfrak{z}_{1}$ is determined uniquely, up to conjugation in $\mathfrak{z}_{1}$, by the embedding $\left.\varphi\right|_{\mathfrak{k}_{2}}$. Furthermore, if the subalgebra $\mathfrak{a}_{2} \subset \mathfrak{g}$ contains no simple ideals of the types listed above, then the embedding $\left.\varphi\right|_{\mathfrak{h}_{1}}: \mathfrak{h}_{1} \hookrightarrow \mathfrak{a}_{2}$ is also determined uniquely by the embedding $\left.\varphi\right|_{\mathfrak{k}_{1}}$. Thus, we see that under our assumptions the linear equivalence class of the embedding $\varphi$ coincides with the equivalence class. We now prove that if the last assumption does not hold, then the subalgebra $\mathfrak{a}_{2}$ must be regular. Indeed, it is sufficient to consider only the case $\mathfrak{s o}_{8} \subset E_{8}$ : in the other cases this follows from the classification of the simple subalgebras. But it follows from the same classification (see [5]) that only in the case of the non-regular subalgebra $\mathfrak{s o}_{8} \subset E_{7} \subset E_{8}$ do we have $\operatorname{dim} \mathfrak{z}_{\mathfrak{g}}\left(\mathfrak{s o}_{8}\right) \geq 3$ (number 24 in Table 8 ). Here $\mathfrak{z}_{\mathfrak{g}}\left(\mathfrak{s o}_{8}\right)=A_{1}$, but $\mathfrak{z}_{\mathfrak{g}}\left(A_{1}\right)=E_{7} \neq \mathfrak{s o}_{8}$.

Proposition 9. Let $\varphi$ be an interesting embedding. Then in the algebra $\mathfrak{h}$ there exists a proper ideal $\mathfrak{h}_{1} \subset \mathfrak{h}$ such that either the subalgebra $\mathfrak{a}_{1} \subset \mathfrak{g}$ or $\mathfrak{z}_{1} \subset \mathfrak{g}$ contains exactly one of the subalgebras $D_{4}, D_{5}, D_{6}, E_{6}, E_{7}$ as a simple ideal.

Proof. The only thing that remains to be proved, in the light of our discussion, is why the subalgebra $D_{7} \subset \mathfrak{g}=E_{8}$ is missing from the list indicated in the statement. Clearly, it is not contained in the centralizer of any semisimple subalgebra of $\mathfrak{g}$. Moreover, $\mathfrak{z}_{\mathfrak{g}}\left(D_{7}\right) \simeq$ $T_{1}$. Therefore it is sufficient to consider the case where $\mathfrak{z}_{\mathfrak{g}}\left(D_{7}\right)$ is a Cartan subalgebra of some simple three-dimensional subalgebra $\mathfrak{s} \subset \mathfrak{g}$. We obtain from the classification of characteristics that there exist two subalgebras $\mathfrak{s}_{1}, \mathfrak{s}_{2}$ with this property. We state an auxiliary result whose proof follows immediately from the definitions.

Lemma 3. For a given uninteresting subalgebra $\mathfrak{h} \subset \mathfrak{g}$ to be an ideal of some interesting subalgebra of $\mathfrak{g}$ it is necessary and sufficient that there exist two embeddings into $\mathfrak{z}_{\mathfrak{g}}(\mathfrak{h})$ that are linearly equivalent in $\mathfrak{z}_{\mathfrak{g}}(\mathfrak{k})$ (where $\mathfrak{k} \subset \mathfrak{h}$ is a Cartan subalgebra) and are not taken to one another by any element of the group $Z_{G}(\mathfrak{h})$.

Using [1,

$$
Z_{G}\left(\mathfrak{s}_{1}\right)=\operatorname{Spin}_{13}, \quad Z_{G}\left(\mathfrak{s}_{2}\right)=\mathbb{Z}_{2} \curlywedge\left(G_{2} \times G_{2}\right) .
$$

In this case the subalgebras $\mathfrak{s}_{i}, i=1,2$, cannot be ideals of interesting subalgebras. Indeed, linearly equivalent embeddings into $\mathfrak{s o}_{14}$ contained in $\mathfrak{s o}_{13}$ are also linearly equivalent in $\mathfrak{s o}_{13}$, which explains the first case. In the second case there are few candidates for interesting embeddings, since the maximal semisimple subalgebras in $G_{2}$ are $A_{2}$ and $A_{1}+A_{1}$. An easy inspection of all possibilities proves the proposition.

Using Theorem 4 one can easily find all the pairs of linearly equivalent embeddings into $\mathfrak{s o}_{8}, \mathfrak{s o}_{10}$ and $\mathfrak{s o}_{12}$ that are not equivalent. In the first two cases these are listed in Example 1. In the last case there are exactly two pairs of such embeddings. The first pair is defined by the embedding ad $\otimes N \otimes N+N \otimes T \otimes T$ of the algebra $\mathfrak{a}=$ $\mathfrak{s l}_{3}+\mathfrak{s l}_{2}+\mathfrak{s l}_{2}$, where $N$ and $T$ are the trivial and tautological representations of the 
algebra $\mathfrak{s l}_{2}$, respectively. The second pair is defined by the restriction of this embedding to the subalgebra formed by the first two ideals of the algebra $\mathfrak{a}$.

We consider separately two cases: case D (case E) where either $\mathfrak{z}_{1}$ or $\mathfrak{a}_{2}$ contains as a simple ideal one of the subalgebras $D_{4}, D_{5}, D_{6}$ (respectively, $E_{6}, E_{7}$ ) of the algebra $\mathfrak{g}$. We call the corresponding subalgebras $\mathfrak{h} \subset \mathfrak{g}$ subalgebras of type D or E.

4.3. Case D. From the description of the linearly equivalent but not equivalent embeddings into $\mathfrak{s o}_{8}, \mathfrak{s o}_{10}$ and $\mathfrak{s o}_{12}$ given above, we see that among the simple ideals of the algebra $\mathfrak{h}$ there is one of type $A_{2}$ or $B_{2}$. In the first case its image under the embedding $\varphi$ is contained in $D_{4}$ or in $D_{4}+A_{2}$, and in the second case, in $D_{5}$. Since the S-subalgebra $\mathfrak{s l}_{3} \subset D_{4}+A_{2}$ (for $\mathfrak{g}=E_{8}$ ) has a two-dimensional torus as its centralizer in $\mathfrak{g}$, the case of embedding into $D_{4}+A_{2}$ is impossible (recall that we are considering only non-simple subalgebras $\mathfrak{h} \subset \mathfrak{g})$.

Thus, we can assume that $\mathfrak{h}_{1}=\mathfrak{s l}_{3} \subset D_{4}$ or $\mathfrak{h}_{1}=\mathfrak{s o}_{5} \subset D_{5}$ (in both cases the embedding is defined by the adjoint representation). We need to find all those pairs of linearly equivalent embeddings into $\mathfrak{z}_{1}$ that are not taken to one another by an element of the group $Z_{G}\left(\mathfrak{h}_{1}\right)$ (see Lemma 3). Clearly, we can immediately discard the variants of those exceptional algebras $\mathfrak{g}$ for which the rank of the semisimple part of the subalgebra $\mathfrak{a}_{1} \subset \mathfrak{g}$ is at most 1 .

The case $\mathfrak{h}_{1}=\mathfrak{s l}_{3}$. Using Dynkin's classification of the simple subalgebras we can find the dimensions of the subalgebras $\mathfrak{a}_{1}$ and $\mathfrak{z}_{1}$ for each exceptional algebra $\mathfrak{g}$, after which it is easy to guess these subalgebras. We give the corresponding Table 29

TABLE 2. $\mathfrak{h}_{1}=\mathfrak{s l}_{3}$

\begin{tabular}{|c|c|c|c|}
\hline $\mathfrak{g}$ & $E_{6}$ & $E_{7}$ & $E_{8}$ \\
\hline $\mathfrak{a}_{1}$ & $T_{2}$ & $3 A_{1}$ & $D_{4}$ \\
\hline $\mathfrak{z}_{1}$ & & $A_{5}+T_{2}$ & $E_{6}+T_{2}$ \\
\hline
\end{tabular}

It is clear from this table that the case $\mathfrak{g}=E_{6}$ is impossible. Let $\mathfrak{g}=E_{7}$. In the next section we will show that $Z_{G}\left(\mathfrak{h}_{1}\right)=\mathbb{Z}_{3}<\left(\mathrm{SL}_{2} \times \mathrm{SL}_{2} \times \mathrm{SL}_{2}\right)$. It is easy to see that we have interesting embeddings of the algebras $\mathfrak{h}=\mathfrak{h}_{1}+\mathfrak{h}_{2}$ only for $\mathfrak{h}_{2}=2 A_{1}$ and $\mathfrak{h}_{2}=3 A_{1}$, and there is a partition into exactly two equivalence classes in each case (they differ by a transposition of the simple ideals of the subalgebra $\mathfrak{h}_{2}$ ).

We finally consider the case $\mathfrak{g}=E_{8}$. In the next section we will prove that $Z_{G}\left(\mathfrak{h}_{1}\right)=$ $\mathbb{Z}_{3} \curlywedge \operatorname{Spin}_{8}$. It then follows from Theorem 6 and Lemma 3 that every interesting subalgebra of the form $\mathfrak{h}=\mathfrak{h}_{1}+\mathfrak{h}_{2}$ must satisfy the following condition: the embedding $\varphi_{2}: \mathfrak{h}_{2} \hookrightarrow D_{4}\left(\sim \mathfrak{z}_{\mathfrak{g}}\left(D_{4}\right)\right)$ is such that $\sigma \circ \varphi_{2} \nsim \tau \circ \varphi_{2}$, where $\sigma$ is an outer involution of the algebra $D_{4}$, and $\tau \in \mathbb{Z}_{3}<\operatorname{Int} D_{4}$. We call such subalgebras $\mathfrak{h}_{2} \subset D_{4}$ even. They are characterized by the fact that no outer automorphism of the algebra $D_{4}$ takes these subalgebras to subalgebras acting on a non-degenerate odd-dimensional subspace. Indeed, if the algebra $\mathfrak{h}_{2}$ normalizes such a subspace, then $\sigma \circ \varphi_{2} \sim \varphi_{2}$. Conversely, if there exists an outer automorphism (whose Kac diagram has index 2) of the algebra $D_{4}$ centralizing $\mathfrak{h}_{2}$, then $\mathfrak{h}_{2}$ is contained in one of the subalgebras $\mathfrak{s o}_{7}, \mathfrak{s o}_{3}+\mathfrak{s o}_{5}$ (see, for example, [6]). Hence the role of $\mathfrak{h}_{2}$ can be played only by four subalgebras: $D_{4}, 4 A_{1}, 3 A_{1}$, and $\mathfrak{s l}_{3}$ (the last one is embedded into $D_{4}$ via its adjoint representation). Obviously, each of the corresponding linear equivalence classes contains exactly two equivalence classes. 
TABLE $3 . \mathfrak{h}_{1}=\mathfrak{s o}_{5}$

\begin{tabular}{|c|c|c|c|}
\hline $\mathfrak{g}$ & $E_{6}$ & $E_{7}$ & $E_{8}$ \\
\hline $\mathfrak{a}_{1}$ & $T_{1}$ & $A_{1}+T_{1}$ & $A_{3}$ \\
\hline $\mathfrak{z}_{1}$ & & & $D_{4}+T_{4}$ \\
\hline
\end{tabular}

The case $\mathfrak{h}_{1}=\mathfrak{s o}_{5}$. In this subsection we use Table 3 .

It is clear that only the case $\mathfrak{g}=E_{8}$ is of interest. Note that $Z_{G}\left(\mathfrak{h}_{1}\right)=\mathrm{SL}_{4}$. Since in the algebra $D_{4}$ a Weyl involution is an inner automorphism, we obtain that the subalgebras $\mathfrak{h}=\mathfrak{h}_{1}+\mathfrak{h}_{2}, \mathfrak{h}_{2}=A_{3}$, or $\mathfrak{h}_{2}=A_{2}$, and only these, are interesting and contain $\mathfrak{h}_{1}$ as a simple ideal. Here the corresponding linear equivalence classes are partitioned into two classes of equivalent embeddings that differ by an outer automorphism of the algebra $\mathfrak{h}$.

The classification obtained above implies immediately that there are no non-simple interesting embeddings into $E_{6}$. Consequently, any two linearly equivalent non-equivalent embeddings into $E_{6}$ are taken to one another by an outer automorphism of the algebra $E_{6}$.

4.4. Case E. Since $Z_{E_{7}}\left(E_{6}\right)=T_{1}$, it is sufficient to examine the cases where the subalgebra $\mathfrak{h}_{1}$ is a simple three-dimensional subalgebra, such that it has a torus with centralizer of type $E_{6}$. From the classification of characteristics we find that there is exactly one candidate for $\mathfrak{h}_{1}$, and by [1, $Z_{E_{7}}\left(\mathfrak{h}_{1}\right)=F_{4}$. Thus, in the case $\mathfrak{g}=E_{7}$ there are no interesting subalgebras of type $\mathrm{E}$ (Theorems 6, 3).

We have obtained a classification of the interesting embeddings into $E_{7}$. In contrast to the case $\mathfrak{g}=E_{6}$, there are no simple interesting embeddings here, but there are two semisimple ones.

We now consider the algebra $\mathfrak{g}=E_{8}$. All the simple three-dimensional subalgebras $\mathfrak{s}_{i} \subset \mathfrak{g}$ whose characteristics $h_{i}$ have centralizers with one of the types $E_{6}, E_{6}+A_{1}$ or $E_{7}$ (modulo the centers) are listed in Table 4 (note that the group $\operatorname{Spin}_{5}$ indicated in the table is contained in the regular subgroup $\left.\mathrm{SL}_{4} \subset G\right)$.

TABLE 4. The centralizers of three-dimensional subalgebras and their characteristics

\begin{tabular}{|c|c|c|c|c|c|}
\hline$i$ & 1 & 2 & 3 & 4 & 5 \\
\hline $\mathfrak{z}_{\mathfrak{g}}\left(h_{i}\right)$ & $E_{7}+T_{1}$ & $E_{7}+T_{1}$ & $E_{6}+A_{1}+T_{1}$ & $E_{6}+A_{1}+T_{1}$ & $E_{6}+T_{2}$ \\
\hline$Z_{G}\left(\mathfrak{s}_{i}\right)$ & $\widehat{E_{7}}$ & $\mathbb{Z}_{2} \wedge \widehat{E_{6}}$ & $F_{4} \times \mathrm{SL}_{2}$ & $\operatorname{Spin}_{5} \times \mathrm{SL}_{2}$ & $F_{4}$ \\
\hline
\end{tabular}

From Theorem 6 and the classification of the interesting embeddings into $E_{6}, E_{7}$ that is already available, we obtain only one series of interesting subalgebras $\mathfrak{h}=\mathfrak{h}_{1}+\mathfrak{h}_{2}$ in $\mathfrak{g}$, where $\mathfrak{h}_{1} \simeq \mathfrak{s l}_{2}: \mathfrak{h}_{1}=A_{1}(i=1)$ and $\mathfrak{h}_{2}$ is an interesting subalgebra in $E_{7}$.

It remains to consider the case $\operatorname{rk} \mathfrak{h}_{1}=2, \mathfrak{z}_{\mathfrak{g}}\left(\mathfrak{k}_{1}\right)=E_{6}+T_{2}$. We can assume the algebra $\mathfrak{h}_{1}$ is simple, that is, of one of the types $A_{2}, B_{2}, G_{2}$. Clearly, the principal three-dimensional subalgebra $\mathfrak{s} \subset \mathfrak{h}_{1}$ must be represented in Table 4 . The indices of the subalgebras $\mathfrak{s}_{i} \subset \mathfrak{g}, i=1, \ldots, 5$, are equal to $1,3,4,12,28$, respectively (see [5]), while the indices of the principal three-dimensional subalgebras $\mathfrak{s}$ can be equal to $4 k, 10 k, 28 k$, $k \in \mathbb{N}$ in the cases $\mathfrak{h}_{1} \simeq \mathfrak{s l}_{3}, \mathfrak{s o}_{5}, G_{2}$, respectively. Therefore we only need to consider the cases where $\mathfrak{h}_{1}$ is the subalgebra $\mathfrak{s l}_{3} \subset \mathfrak{g}$ of index 1 or 3 (index 7 is impossible by [5]), or $\mathfrak{h}_{1}=G_{2} \subset F_{4} \subset \mathfrak{g}$. In the last case we have $\mathfrak{z}_{\mathfrak{g}}\left(\mathfrak{k}_{1}\right)=\mathfrak{z}_{\mathfrak{g}}\left(A_{2}\right)=E_{6}$; this case is of no interest by Theorem 6 (since $\left.\mathfrak{z}_{\mathfrak{g}}\left(G_{2}\right)=F_{4}\right)$. Now suppose that $\mathfrak{h}_{1} \simeq \mathfrak{s l}_{3}$ is a subalgebra of

${ }^{9}$ We leave those squares that yield us no useful information blank. 
index 3. Then $\mathfrak{z}_{\mathfrak{g}}\left(\mathfrak{k}_{1}\right)=E_{6}$ only if $\mathfrak{h}_{1} \subset D_{4}$ is an S-subalgebra, which fact follows from 5 , Table 25]. We have already considered this case above. Finally, let $\mathfrak{h}_{1}=A_{2}$. Then $\mathfrak{z}_{\mathfrak{g}}\left(\mathfrak{h}_{1}\right)=\mathfrak{z}_{\mathfrak{g}}\left(\mathfrak{k}_{1}\right)=E_{6}$. Consequently, in this case we obtain one more series of interesting subalgebras $\mathfrak{h}=\mathfrak{h}_{1}+\mathfrak{h}_{2}: \mathfrak{h}_{1}=A_{2}$ and $\mathfrak{h}_{2} \subset E_{6}$ is an interesting subalgebra in $E_{6}$.

This completes the classification of the interesting subalgebras of exceptional Lie algebras.

4.5. The main result. We summarize all of our preceding discussion.

Theorem 7. Let $\mathfrak{g}$ be an exceptional Lie algebra, and $\mathfrak{h}$ a semisimple Lie algebra. Then the linear equivalence class of an arbitrary embedding $\varphi: \mathfrak{h} \hookrightarrow \mathfrak{g}$ consists, as a rule, of a single class of equivalent embeddings. All the exceptions are listed in Table 9 ,

Let us explain the notation in Table 9 $\varphi^{L}=\left\{\varphi_{1}, \ldots, \varphi_{k}\right\}$ denotes a set of representatives of the classes of equivalent embeddings for a given linear equivalence class of the embedding $\varphi=\varphi_{1}$. When describing the embedding $\varphi_{1}$ we indicate a regular subalgebra $\mathfrak{r}$ with respect to which $\varphi_{1}$ is an S-embedding. The only exceptions are the cases $\mathfrak{h}=\mathfrak{h}_{0}+\mathfrak{s l}_{3} \subset E_{8}$, where $\mathfrak{h}_{0}$ is either an interesting subalgebra of $E_{6}$ or an even subalgebra of $D_{4}$. An arbitrary outer automorphism of order 2 of the algebra $\mathfrak{r}$ is denoted by $\sigma$, and of the algebra $\mathfrak{h}$ by $\tau$. The other notation is self-evident.

Corollary 3. Let $\mathfrak{h}$ be a semisimple subalgebra of a simple exceptional Lie algebra $\mathfrak{g}$. Then the linear conjugacy class of the subalgebra $\mathfrak{h}$ coincides with its conjugacy class in the case $\mathfrak{g}=E_{7}$. In the other cases there are exceptions, which are listed below:

(1) $\mathfrak{g}=E_{6}: \mathfrak{h}_{1}=\mathfrak{s l}_{3}, G_{2}, \mathfrak{s o}_{5}$ are $S$-subalgebras in $E_{6}, E_{6}, D_{5}$, respectively, and $\mathfrak{h}_{2}=\sigma\left(\mathfrak{h}_{1}\right)$;

(2) $\mathfrak{g}=E_{8}: \mathfrak{h}_{1}=\mathfrak{s l}_{3}$ is an $S$-subalgebra in $E_{6}+A_{2}$ and $\mathfrak{h}_{2}=(\sigma \times \mathrm{Id})\left(\mathfrak{h}_{1}\right)$.

Here $\mathfrak{h}_{i}, i=1,2$, denote representatives of the conjugacy classes of one linear conjugacy class and $\sigma$ is an outer involution of the corresponding simple Lie algebra.

Remark 4. Dynkin [5] found three (of the four) cases of partitions of linear conjugacy classes. Namely, two of them are S-subalgebras in $E_{6}$, and the third case is the S-subalgebra $\mathfrak{s l}_{3} \subset D_{4}+D_{4} \subset E_{8}$. However, in the last case the number of conjugacy classes in the partition was not indicated. According to Corollary 3. there are exactly two such classes in each of these cases.

\section{NORMALIZERS OF SIMPLE SUBALGEBRAS}

In this section we find the normalizers of the simple subalgebras $\mathfrak{h}$ of rank greater than 1 in the adjoint exceptional Lie groups $G$; more precisely, the groups $\Gamma \wedge Z_{G}(\mathfrak{h})$, where the groups $\Gamma \simeq \Gamma(\mathfrak{h}) \subset$ Aut $\mathfrak{h} /$ Int $\mathfrak{h}$ were calculated above. From the results of $\S 2$, it follows that in all cases the group of realizable outer automorphisms of the algebra $\mathfrak{h}$ embeds into $N_{G}(\mathfrak{h})$, that is, $N_{G}(\mathfrak{h})=\Gamma \curlywedge\left(H \cdot Z_{G}(\mathfrak{h})\right)$. First we find the groups $Z_{G}(\mathfrak{h})$; this occupies the larger part of this section. Then we determine the action of the group $\Gamma$ on the group $Z_{G}(\mathfrak{h})$. Thus we shall find the groups $\Gamma<Z_{G}(\mathfrak{h})$.

The centralizers of the simple three-dimensional subalgebras were calculated by Alekseevskiı [1]. The method proposed in that paper is also suitable in our case. We present this method in a form convenient for our purposes.

5.1. Alekseevskiı̌'s results. Let $G$ be the adjoint semisimple Lie group, and $\mathfrak{h}$ a semisimple subalgebra of a Lie algebra $\mathfrak{g}$, and let $\mathcal{R}(\mathfrak{h})^{S}=\left\{\mathfrak{r}_{1}, \ldots, \mathfrak{r}_{k}\right\}$ be the set of regular subalgebras, considered up to conjugacy in $\mathfrak{g}$, in which $\mathfrak{h}$ is an S-subalgebra.

Suppose that every subalgebra $\mathfrak{r}_{i} \in \mathcal{R}(\mathfrak{h}), i=1, \ldots, k$, satisfies condition C: If two embeddings $\mathfrak{h} \hookrightarrow \mathfrak{r}_{i}$ are equivalent in $\mathfrak{g}$, then they are taken to one another by an element 
of $N_{G}\left(\mathfrak{r}_{i}\right)$. This condition is satisfied in the case where the algebra $\mathfrak{h}$ is isomorphic to $\mathfrak{s l}_{2}$. This can be seen from Dynkin's classification of the $\mathfrak{s l}_{2}$-subalgebras [5, Theorems 9.2, 9.3]: three-dimensional S-subalgebras of the same index in a simple Lie algebra are conjugate. If the algebra $\mathfrak{h}$ is simple and $\mathfrak{g}$ is an exceptional Lie algebra, then condition $\mathrm{C}$ is also satisfied, except for two cases. This follows from the classification of the simple embeddings (for the cases $G_{2}$ and $F_{4}$ see the first two columns of Tables 10 and 11). The exceptional cases are $\mathfrak{g}=E_{7}$ or $E_{8}, \mathfrak{h}=\mathfrak{s o}_{8} \subset A_{7} \subset E_{7}$.

Let $R_{i}$ be a connected regular subgroup of $G$ with tangent algebra $\mathfrak{r}_{i}$, and let $\overline{R_{i}}$ be a subgroup of maximal rank with semisimple part $R_{i}$. We consider the set $\mathcal{D}(\mathfrak{h})=$ $\left\{D_{1}, \ldots, D_{k}\right\}$, where $D_{i}=Z\left(\overline{R_{i}}\right)$. It follows from condition $\mathrm{C}$ that the conjugacy classes of the subalgebras $\mathfrak{r}_{i} \subset \mathfrak{g}$ containing $\mathfrak{h}$ are in a one-to-one correspondence with the conjugacy classes of the subgroups $D_{i} \subset Z=Z_{G}(\mathfrak{h})$. The subgroups in $\mathcal{D}(\mathfrak{h})$ are characterized by the fact that these are the maximal quasitor 10 in $Z$, up to conjugacy in $Z$, that are regular in $G$ (that is, contained in some maximal torus of the group $G$ ). Note that a subgroup of a simply connected group that is isomorphic to $\mathbb{Z}_{n} \times \mathbb{Z}_{m}$ is regular. This follows from the fact that every semisimple element of a simply connected group has a connected centralizer (see, for example, [3]).

A complete set of invariants of the reductive group $Z$ is as follows:

(1) the Lie algebra $\mathfrak{z}$ of the group $Z$;

(2) the fundamental group $F$ of the semisimple part $Z^{s}$ of the group $Z^{\circ}$;

(3) the group $P=Z^{s} \cap C^{\circ}$, where $C$ is the center of the group $Z$;

(4) the group of components $K=Z / Z^{\circ}$;

(5) the extension class $\omega$ of the group $Z^{\circ}$ by $K$.

In most cases the group $F$ can be found by using the following assertion.

Proposition 10. Suppose that none of the groups $D_{i} / D_{i}^{\circ}$ contains a subgroup of the form $\mathbb{Z}_{p} \times \mathbb{Z}_{p}$, where $p$ is a prime divisor of the order of the center of the group $\widehat{Z^{s}}$. If the group $G$ is simply connected, then the group $Z^{s}$ is also simply connected. In any case, $Z^{s} \simeq \widehat{Z^{s}} / S$, where the group $S$ is isomorphic to a subgroup of the center of the simply connected covering $\widehat{G}$ of the group $G$.

In the case where the group $G$ is simply connected, the group $Z^{s}$ is simply connected for most of the subalgebras $\mathfrak{h}$; this can be proved by applying Proposition [10. In the remaining cases the group $F$ can also be found merely from the description of the group $D_{i}$. For a non-simply connected group $G$ the method of embedding into a smaller subgroup is used. This method consists in passing from the group $G$ to the subgroup $Z_{G}(Y)$, where $Y$ is some subgroup of the center of the group $Z^{\circ}$. Clearly, $\mathfrak{z}_{\mathfrak{g}}(Y) \supset \mathfrak{h}$. The same method is used for finding the group $P$ : in this case we set $Y=C^{\circ}$. We will illustrate this in subsequent examples. It is also useful to bear in mind that the derived subgroups of complete regular subgroups of simply connected groups are simply connected (see, for example, [3]).

Let $\tau: K \rightarrow$ Aut $Z^{\circ} /$ Int $Z^{\circ}$ be the canonical homomorphism, and $K_{0}$ its kernel. The following propositions easily follow from the characterization of the subgroups $D_{i} \subset Z$.

Proposition 11. The group $K_{0}$ is trivial if and only if among the groups $D_{i} \in \mathcal{D}(\mathfrak{h})$ there exists a connected group of maximal dimension.

Proposition 12. If all the groups $D_{i}$ have the same dimension, then $K=K_{0}$.

\footnotetext{
${ }^{10} \mathrm{~A}$ quasitorus is a commutative algebraic group consisting of semisimple elements. Such group are also called diagonalizable.
} 
Proposition 13. Let $\pi: Z \rightarrow K$ be the natural projection. If $D_{i} / D_{i}^{\circ}$ is a non-trivial cyclic group, then $\pi\left(D_{i}\right) \neq 1$. Furthermore, every element of the group $K$ is conjugate in $K$ to an element of one of the subgroups $\pi\left(D_{i}\right)$.

The extension class $\omega$ is determined by an element of the group $H^{2}(K, C)$. In the cases $K \neq 1, C \neq 1$ the groups $K$ are fairly small $\left(\mathbb{Z}_{2}, \mathbb{Z}_{3}, \mathbb{V}_{4}\right.$, and $\left.\mathbb{S}_{3}\right)$; therefore the cohomology groups easily can be calculated. They are as a rule trivial. In the other cases one can use the method of embedding into a smaller subgroup. As a result we obtain that $\omega=1$ in all cases.

5.2. Finding the groups $\boldsymbol{N}=\boldsymbol{\Gamma} \wedge \boldsymbol{Z}$. We make several remarks, which simplify the calculations. Some examples of their application will be considered at the end of the section.

As a rule, if the group $\Gamma$ is non-trivial, then it is isomorphic to $\mathbb{Z}_{2}$ (the only possible exception is if the algebra $\mathfrak{h}$ is of type $D_{4}$ ). Therefore we actually need to find the action on $Z$ of only one element.

In many cases an outer automorphism $\sigma$ of the subalgebra $\mathfrak{h}$ is realized by some Weyl involution $\theta$ of the algebra $\mathfrak{g}$ normalizing some regular subalgebra $\mathfrak{r}_{1} \in \mathcal{R}(\mathfrak{h})^{S}$. Then $\theta$ acts by inversion on the corresponding group $D_{1} \subset Z$. If the automorphism $\sigma$ is actually realized in $\mathfrak{r}_{1}$, then the corresponding element of the group $\Gamma$ commutes with $D_{1}$. By using these considerations it is often possible to determine the group $N$.

We observe that if $Z_{G}(\mathfrak{h})=Z_{L}(\mathfrak{h}) \cdot Z_{G}(L)$ is an almost direct product, where $H \subset$ $L \subset G$, and the outer automorphisms of the subalgebra $\mathfrak{h}$ are realized in $\mathfrak{l}$, then $\Gamma \wedge Z=$ $\left(\Gamma<Z_{L}(\mathfrak{h})\right) \cdot Z_{G}(L)$.

A great deal (if not all) of information about the group $N$ can be extracted from the group $Z_{0}=Z_{G}\left(\mathfrak{h}^{\Gamma}\right)$. This follows from the fact that $N \subset Z_{0}$.

If there is only one regular subalgebra of minimal rank $\mathfrak{r} \in \mathcal{R}(\mathfrak{h})^{S}$ and it is complete, which practically always holds, then an element $\sigma \in \Gamma$ acts as an inner automorphism on the group $Z^{\circ}$ if and only if the automorphism of the subalgebra $\mathfrak{h}$ corresponding to $\sigma$ is realized in $\mathfrak{r}$. This follows from the fact that the centralizer of a maximal torus of the group $Z^{\circ}$ is a connected regular subgroup with tangent algebra $\mathfrak{r}$ (if the assumptions about the subalgebra $\mathfrak{r}$ are not satisfied, then it should be replaced by $\tilde{\mathfrak{r}}$ from Proposition 3 ).

Note that for some subalgebras $\mathfrak{h}$ (namely, for $\mathfrak{h}=A_{2}^{1} \subset E_{6}, \mathfrak{h}=D_{4}^{2} \subset E_{8}$ ) the group $N$ was actually found in $\S 2$.

5.3. Description of Tables 10-14. In these tables, $\mathfrak{h} \subset \mathfrak{g}$ is a simple subalgebra of an exceptional Lie algebra $\mathfrak{g}$. Recall that in the cases $\mathfrak{g}=G_{2}, F_{4}, E_{8}$ the group $G=\operatorname{Int} \mathfrak{g}$ is simply connected, and in the cases $\mathfrak{g}=E_{6}, E_{7}$ its fundamental group is isomorphic to $\mathbb{Z}_{3}, \mathbb{Z}_{2}$, respectively.

The notation for the subalgebras $\mathfrak{h}$ is taken from [5] (that is, we indicate the type and index, possibly with primes). We use this notation because it agrees with the notation in [1]. We denote this way subalgebras considered up to linear conjugacy. According to our classification of subalgebras up to conjugacy, for the subalgebras whose linear conjugacy classes are partitioned we indicate the corresponding number in parentheses.

In the second and third column we present the sets $\mathcal{R}(\mathfrak{h})^{S}$ and $\mathcal{D}(\mathfrak{h})$. We know the first from the classification of the simple subalgebras obtained above, while the centers of the subalgebras of maximal rank can be found, for example, in [1]. In the fourth column we indicate the algebras $\mathfrak{z}_{\mathfrak{g}}(\mathfrak{h})$, which can be easily determined since we know their dimensions, which were found in [5].

The fifth and sixth columns contain the main result of this section, namely, in these columns we indicate the groups $Z=Z_{G}(\mathfrak{h})$ and $N=\Gamma \curlywedge Z$. 
First we will explain our notation. By $\left(G_{1} \times G_{2} \times \cdots \times G_{n}\right) / S$ we mean the quotient of the direct product of the groups $G_{1}, \ldots, G_{n}$ by a subgroup isomorphic to $S$ and diagonally embedded into the direct product of the centers of these groups. In all the cases that we encounter such a subgroup is determined uniquely up to an automorphism of the group $G_{1} \times G_{2} \times \cdots \times G_{n}$. The direct product of $n$ copies of the group $G$ is denoted by $G^{n}$. By $G \cdot T$ we mean an almost direct product of a semisimple group $G$ and a torus $T$ in which $G \cap T=Z(G)$, except for the case considered in Example 8 (below). The notation $G_{1} \wedge G_{2}$ always means a semidirect product such that none of the non-trivial elements of the group $G_{1}$ centralizes the group $G_{2}$. Bearing in mind this stipulation, the group $G_{1} \wedge G_{2}$ is, as a rule, uniquely determined up to isomorphism. We now list the exceptions:

(1) $\mathfrak{h}=A_{2}^{1}, \mathfrak{g}=E_{6}, N=\mathbb{Z}_{2} \wedge\left(\left(\mathrm{SL}_{3} \times \mathrm{SL}_{3}\right) / \mathbb{Z}_{3}\right)$, and the group $\Gamma=\mathbb{Z}_{2}$ acts by interchanging the direct factors;

(2) $\mathfrak{h}=A_{3}^{1}, \mathfrak{g}=E_{6}, N=\mathbb{Z}_{2} \curlywedge\left(\left(\mathrm{SL}_{2} \times \mathrm{SL}_{2}\right) \cdot \mathrm{T}_{1}\right)$, and the group $\Gamma=\mathbb{Z}_{2}$ acts by interchanging the factors $\mathrm{SL}_{2}$;

(3) $\mathfrak{h}=A_{4}^{1}, \mathfrak{g}=E_{7}, N=\mathbb{Z}_{2} \curlywedge\left(\mathrm{SL}_{3} \cdot \mathrm{T}_{1}\right)$, and the non-trivial element of the group $\Gamma=$ $\mathbb{Z}_{2}$ acts as a Weyl involution on $Z$.

5.4. Examples of finding the group $Z=Z_{G}(\mathfrak{h})$.

Example 3. We consider the subalgebra $\mathfrak{h}=A_{2}^{3^{\prime}}$ of the algebra $\mathfrak{g}=F_{4}$. In this case, $D_{1} \simeq \mathbb{V}_{4}, D_{2} \simeq \mathbb{Z}_{3}$ (recall that the maximal diagonalizable subgroups $D_{i} \in \mathcal{D}(\mathfrak{h})$ are the characteristics of minimal regular subalgebras containing $\mathfrak{h}$; they are known for all regular subalgebras). Consequently, the group $Z$ is finite and contains, apart from the identity element, only elements of order 2 and 3 (since every finite cyclic subgroup is regular). Therefore $Z=S_{2} \cdot S_{3}$, where $S_{p}$ is a Sylow $p$-subgroup, and $S_{2} \simeq \mathbb{Z}_{2}^{k}, k \geq 2$. Since the group $G$ is simply connected, we have $S_{3} \simeq \mathbb{Z}_{3}$; otherwise the group $Z$ would contain a regular subgroup of the form $\mathbb{Z}_{3} \times \mathbb{Z}_{3}$, which is false. Then from the fact that all the subgroups of $Z$ isomorphic to $\mathbb{V}_{4}$ are conjugate we see that there are no more of them in $S_{2}$ than the order of the subgroup $S_{3}$, that is, 3 . Therefore, $k=2$. Next, the number of Sylow 2 -subgroups in $Z$ is equal to either 1 or 3 . We claim that the second case is impossible. Assume the opposite and consider the natural homomorphism of permuting the Sylow 2subgroups $\varphi: Z \rightarrow \mathbb{S}_{3}$. This homomorphism is obviously surjective (otherwise its kernel would be a Sylow 2-subgroup, which cannot be contained in the normalizer of another Sylow 2-subgroup). Therefore, $\operatorname{Ker} \varphi \simeq \mathbb{Z}_{2}$. Hence we obtain the existence of an element of order six in the group $Z$, which is impossible. Thus, $Z \simeq \mathbb{Z}_{3} \wedge \mathbb{V}_{4} \simeq \mathbb{A}_{4}$.

Example 4. Let $\mathfrak{g}=F_{4}$ and $\mathfrak{h}=G_{2}^{1}$. We have $D_{1} \simeq \mathrm{T}_{1}, D_{2} \simeq \mathbb{V}_{4}, \mathfrak{z} \sim A_{1}^{8}$. We now find the group $K$. By Proposition 11 this group acts by outer automorphisms on $Z^{\circ}$. But Aut $\mathfrak{s l}_{2}=\operatorname{Int} \mathfrak{s l}_{2}$; therefore the group $K$ is trivial. Consequently, the group $Z$ is connected. Since the group $\mathrm{SL}_{2}$ has no subgroups isomorphic to $\mathbb{V}_{4}$, we obtain that $Z \simeq$ $\mathrm{SO}_{3}$.

Example 5. We consider the case $\mathfrak{g}=E_{6}, \mathfrak{h}=A_{2}^{1}=A_{2}, D_{1} \simeq \mathrm{T}_{4}, \mathfrak{z} \sim 2 A_{2}^{1}$ (where $\sim$ denotes conjugacy in $\mathfrak{g}$ ). It follows from Proposition 13 that the group $Z$ is connected. Therefore we only need to find the group F. According to Proposition 10 it is either trivial or isomorphic to $\mathbb{Z}_{3}$. We prove by contradiction that the first case is impossible. Note that the simple ideals of the subalgebra $\mathfrak{z}$ are conjugate to $\mathfrak{h}$. Therefore a connected regular subgroup of $G$ with Lie algebra $3 A_{2}$ is simply connected, which contradicts the well-known fact that its center is isomorphic to $\mathbb{Z}_{3}$. Therefore $Z \simeq\left(\mathrm{SL}_{3} \times \mathrm{SL}_{3}\right) / \mathbb{Z}_{3}$, where the subgroup $\mathbb{Z}_{3} \subset Z\left(\mathrm{SL}_{3}\right) \times Z\left(\mathrm{SL}_{3}\right)$ is embedded diagonally.

Example 6. Consider the case $\mathfrak{g}=E_{6}, \mathfrak{h}=G_{2}^{1}, D_{1} \simeq \mathrm{T}_{2}, \mathfrak{z} \sim A_{2}^{2^{\prime \prime}}$. Clearly, the group $Z$ is connected. We find its fundamental group $F$. If this group were trivial, 
then the subalgebra $G_{2}^{1}+A_{2}^{2^{\prime \prime}}$ would be an R-subalgebra (since it would have non-trivial centralizer in $G$ ). But it follows from Dynkin's classification that this is an S-subalgebra. Therefore, $Z \simeq \mathrm{PSL}_{3}$.

Example 7. Consider the case $\mathfrak{g}=E_{6}, \mathfrak{h}=B_{2}^{1}, D_{1} \simeq \mathrm{T}_{3}, \mathfrak{z} \sim B_{2}^{1}+T_{1}$. By Propositions 11 and 12 the group $Z$ is connected and by Proposition 10 the group $F$ is trivial (since the group $\mathbb{Z}_{2} \simeq Z\left(\mathrm{Sp}_{4}\right)$ does not embed into the group $\mathbb{Z}_{3} \simeq Z\left(\widehat{E_{6}}\right)$ ). We now find the group $P$. We apply the method of embedding into a smaller subgroup. Obviously, the connected center $C^{\circ}$ of the group $Z$ is a one-dimensional torus. Furthermore, $Z_{G}\left(D_{5}^{1}\right)$ is also a one-dimensional torus; moreover, it is conjugate in $\mathfrak{g}$ to the torus $C^{\circ}$, since $2 B_{2}^{1} \subset D_{5}^{1}$. Therefore for the subgroup $\operatorname{Spin}_{10} \subset G$ corresponding to the subalgebra $D_{5}^{1} \supset \mathfrak{h}$ we have $C \cap \operatorname{Spin}_{10}=Z\left(\operatorname{Spin}_{10}\right)$. Then $P=Z\left(\operatorname{Spin}_{10}\right) \cap \operatorname{Spin}_{5} \simeq \mathbb{Z}_{2}$. The last isomorphism follows, for example, from the fact that the image of the group $\operatorname{Spin}_{5}$ under the canonical homomorphism $\pi: \operatorname{Spin}_{10} \rightarrow \mathrm{SO}_{10}$ is isomorphic to $\mathrm{SO}_{5}$. Thus, we obtain $Z \simeq \mathrm{Sp}_{4} \cdot \mathrm{T}_{1} \simeq\left(\mathrm{Sp}_{4} \times \mathrm{T}_{1}\right) / \mathbb{Z}_{2}$

Example 8. Let $\mathfrak{g}=E_{6}, \mathfrak{h}=A_{3}^{1}$. Then $D_{1} \simeq \mathrm{T}_{3}, \mathfrak{z} \sim 2 A_{1}^{1}+T_{1}$. It is clear that in this case the group $Z$ is connected and the group $F$ is trivial. It remains only to find the group $P$. We apply the method of embedding into a smaller subgroup. As in the preceding example, the question reduces to the situation $C^{\circ}=Z_{G}\left(D_{5}^{1}\right) \simeq \mathrm{T}_{1}$ : this can be done, since $\mathfrak{r}=A_{3}+2 A_{1} \subset D_{5}$. In a similar fashion we obtain $P=Z\left(\operatorname{Spin}_{10}\right) \cap \operatorname{Spin}_{4}$ (the subgroup of $\operatorname{Spin}_{10}$ corresponding to the subalgebra $\mathfrak{s l}_{2}+\mathfrak{s l}_{2} \simeq \mathfrak{s o}_{4}$ is simply connected). If $\pi$ is the covering in the preceding example, then $\pi(R) \simeq \mathrm{SO}_{6} \times \mathrm{SO}_{4}=\pi\left(\operatorname{Spin}_{6}\right) \times \pi\left(\operatorname{Spin}_{4}\right)$. Hence it easily follows that $\operatorname{Spin}_{6} \cap \operatorname{Spin}_{4}=\operatorname{Ker} \pi \simeq \mathbb{Z}_{2}$. Moreover, since $Z\left(\mathrm{SO}_{4}\right) \neq$ $Z\left(\mathrm{SO}_{10}\right)$, we obtain that $\operatorname{Ker} \pi=P$. Thus, $Z=\left(\mathrm{SL}_{2} \times \mathrm{SL}_{2}\right) \cdot \mathrm{T}_{1} \simeq\left(\mathrm{SL}_{2} \times \mathrm{SL}_{2} \times \mathrm{T}_{1}\right) / \mathbb{Z}_{2}$, where the group $\mathbb{Z}_{2}$ is generated by an element of order two with non-trivial projections onto the factors.

Example 9. Let us find the centralizer in $G=E_{7}$ of the subalgebra $\mathfrak{h}=A_{2}^{3^{\prime}} \subset \mathfrak{g}$. We have $D_{1} \simeq \mathrm{T}_{3}, D_{2} \simeq \mathbb{Z}_{3} \times \mathrm{T}_{1}, \mathfrak{z} \sim 3 A_{1}^{1}$. According to Propositions[11, 13 we have $K_{0}=1$, $K \simeq \mathbb{Z}_{3}$. It is also clear that the group $K$ acts on the algebra $\mathfrak{z}$ by a cyclic permutation of order three of its simple ideals. Let us find the group $F$. It is either trivial or isomorphic to $\mathbb{Z}_{2}$ (see Proposition (10). But it cannot be trivial because $Z_{G}\left(D_{4}+3 A_{1}\right) \simeq \mathbb{V}_{4}$; see [1]. Therefore $Z=\mathbb{Z}_{3} \wedge\left(\left(\mathrm{SL}_{2} \times \mathrm{SL}_{2} \times \mathrm{SL}_{2}\right) / \mathbb{Z}_{2}\right)$, where $\mathbb{Z}_{2}$ is the diagonally embedded central subgroup.

Example 10. Consider the case $\mathfrak{g}=E_{8}, \mathfrak{h}=B_{3}^{2^{\prime}}, D_{1} \simeq \mathrm{T}_{2}, D_{2} \simeq \mathbb{Z}_{2} \times \mathrm{T}_{1}, D_{3} \simeq \mathbb{V}_{4}$, $\mathfrak{z} \sim A_{1}^{1}+T_{1}$. By Proposition 10 we have $F=1$, and by Proposition 11] $K_{0}=1$. Furthermore, the group $K$ contains an involution. Then it is clear that $K \simeq \mathbb{Z}_{2}$ and the group $K$ acts as an involution on a maximal torus of the group $Z^{\circ}$ (this follows from the fact that the group $Z^{\circ}$ has no other outer automorphisms). To find the group $P$ we observe that $\widehat{E_{7}} \cap Z_{G}\left(E_{7}\right) \simeq \mathbb{Z}_{2}$ ( since $Z_{G}\left(E_{7}\right) \simeq \mathrm{SL}_{2}$ ). The torus $C^{\circ} \simeq \mathrm{T}_{1}$ contains the center of the group $E_{7}$, since this torus is the centralizer in $\widehat{E_{7}}$ of a complete regular subalgebra $A_{6} \subset E_{7}$. Therefore $P \simeq \mathbb{Z}_{2}$ and $Z \simeq \mathbb{Z}_{2} \curlywedge\left(\mathrm{SL}_{2} \cdot \mathrm{T}_{1}\right) \simeq \mathbb{Z}_{2} \curlywedge\left(\mathrm{SL}_{2} \times \mathrm{T}_{1}\right) / \mathbb{Z}_{2}$.

Example 11. Consider the case where condition $\mathrm{C}$ is not satisfied and we cannot use the results of the preceding subsections: $\mathfrak{g}=E_{7}, \mathfrak{h}=D_{4}^{2}, \mathfrak{r}_{1}=A_{7}, D_{1} \simeq \mathbb{Z}_{2}, \mathfrak{z}=0$. The group $Z$ has at most three classes of conjugate subgroups isomorphic to $\mathbb{Z}_{2}$ (because there are exactly three non-equivalent embeddings $\mathfrak{s o}_{8} \hookrightarrow \mathfrak{s l}_{8}$ ). Since all the non-identity elements of the group $Z$ are involutions, we conclude that $Z=\mathbb{Z}_{2}$ or $Z=\mathbb{V}_{4}$. But the first case is impossible, since an outer automorphism of the subalgebra $\mathfrak{r}_{1}$ centralizing $\mathfrak{h}$ is realized in $\mathfrak{g}$, and $Z_{G}\left(\mathfrak{r}_{1}\right) \simeq \mathbb{Z}_{2}$. Therefore, $Z=\mathbb{V}_{4}$. 
We consider the second case where condition $\mathrm{C}$ is not satisfied. We have

$$
\mathfrak{g}=E_{8}, \quad \mathfrak{h}=D_{4}^{2^{\prime}}, \quad \mathfrak{r}_{1}=A_{7}, \quad \mathfrak{r}_{2}=2 D_{4}, \quad D_{1} \simeq \mathbb{Z}_{2} \times \mathrm{T}_{1}, \quad D_{2} \simeq \mathbb{V}_{4}, \quad \mathfrak{z} \sim A_{1}^{1} .
$$

Clearly, $Z \simeq K_{0} \times \mathrm{SL}_{2}$. From the fact that $Z_{G}\left(E_{7}+A_{1}\right) \simeq \mathbb{Z}_{2}$ and from what has already been analyzed above we obtain that $Z \simeq \mathbb{V}_{4} \times \mathrm{SL}_{2}$.

\subsection{Examples of finding the group $N=\Gamma \wedge Z_{G}(\mathfrak{h})$.}

Example 12. Let us prove that in the case of Example 3 we have $N \simeq \mathbb{S}_{4}$. A non-trivial element of the group $\Gamma$ obviously normalizes the subgroup $D_{1} \simeq \mathbb{V}_{4} \subset Z$, but does not centralize it, since an outer automorphism of the subalgebra $\mathfrak{h}$ is realized only by an outer automorphism of the subalgebra $D_{4} \subset \mathfrak{g}$ (note that $\left.D_{1}=Z\left(\operatorname{Spin}_{8}\right) \subset F_{4}\right)$. This clearly implies that $N \simeq \mathbb{S}_{4}$ : every element of the group $N$ that is not contained in $D_{1}$ acts as a non-trivial permutation on the set of three elements of order two in the group $D_{1}$.

Example 13. Let us find the group $N$ for $\mathfrak{h}=A_{3}^{1} \subset F_{4}=\mathfrak{g}$. We have $Z \simeq \mathbb{Z}_{2} \times \mathrm{SL}_{2}$. Let $\sigma \in \Gamma$ be a non-trivial element. Since Aut $\mathfrak{s l}_{2}=\operatorname{Int} \mathfrak{s l}_{2}$, we can assume that $\sigma$ centralizes the subgroup $Z^{\circ} \subset Z$. It only remains to determine the element $\sigma z \sigma^{-1}$, where $1 \neq z \in \mathbb{Z}_{2} \subset Z$. We observe that $\mathfrak{h}^{\sigma} \sim B_{2}^{1}$ and $N \subset Z_{G}\left(B_{2}^{1}\right) \simeq \mathbb{Z}_{2} \wedge\left(\mathrm{SL}_{2} \times \mathrm{SL}_{2}\right)$ (the group $\mathbb{Z}_{2}$ acts by interchanging the direct factors). It is easy to understand the structure of the embedding $N \subset Z_{G}\left(B_{2}^{1}\right)$ : corresponding to the element $\sigma$ is an element of the center of one of the factors of the form $\mathrm{SL}_{2}$. Therefore $\sigma z \sigma^{-1}=z a$, where $a \in Z\left(\mathrm{SL}_{2}\right) \subset N$ is a non-trivial element. Thus, we have described the group $N \simeq \mathbb{Z}_{2} \curlywedge\left(\mathbb{Z}_{2} \times \mathrm{SL}_{2}\right)$.

Using a similar method one can examine the cases $\mathfrak{h}=A_{3}^{2^{\prime}}, A_{3}^{2^{\prime \prime}} \subset E_{7}$.

Example 14. Let us find the group $N$ in the case of Example 8 . Here we have $\Gamma \simeq \mathbb{Z}_{2}$ and $Z \simeq\left(\mathrm{SL}_{2} \times \mathrm{SL}_{2}\right) \cdot \mathrm{T}_{1}$. It is obvious that $Z_{G}\left(C^{\circ}\right) \sim \operatorname{Spin}_{10} \supset H$. An outer automorphism of $\mathfrak{h}$ is realized in $D_{5}$ and interchanges the ideals $\mathfrak{s l}_{2}$ of $\mathfrak{z}_{D_{5}}(\mathfrak{h}) \simeq \mathfrak{s l}_{2}+\mathfrak{s l}_{2}$. Thus, $N \simeq\left(\mathbb{Z}_{2} \curlywedge\left(\mathrm{SL}_{2} \times \mathrm{SL}_{2}\right)\right) \cdot \mathrm{T}_{1}$.

Example 15. Consider the case $\mathfrak{h}=D_{4}^{2} \subset E_{7}=\mathfrak{g}$. We have $Z \simeq \mathbb{V}_{4}$ and $\Gamma \simeq \mathbb{S}_{3}$. Let the group $\Gamma$ be generated by automorphisms (of the algebra $\mathfrak{h}$ ) $\tau$ of order three, and $\sigma$ of order two. We can assume that $\mathfrak{h}^{\tau}=A_{2}^{3^{\prime}}, \mathfrak{h}^{\sigma}=B_{3}^{2}$. From Table 13 we see that $Z_{G}\left(\mathfrak{h}^{\tau}\right) \simeq \mathbb{A}_{4}$ and $Z_{G}\left(\mathfrak{h}^{\sigma}\right) \simeq \mathbb{Z}_{2} \wedge \mathrm{T}_{1} \not \supset \mathbb{Z}_{2} \times \mathbb{V}_{4}$. Consequently, none of the elements of $\Gamma$ centralizes the group $Z$. Therefore, $N \simeq \mathbb{S}_{4}$.

\section{TABLES}

TABLE 5. Restrictions of the simplest representations

\begin{tabular}{|c|c|c|}
\hline $\mathfrak{g}$ & $\mathfrak{r}$ & $\left.\pi_{1}\right|_{\mathfrak{r}}$ \\
\hline \hline$F_{4}$ & $D_{4}$ & $R\left(\pi_{1}\right)+R\left(\pi_{3}\right)+R\left(\pi_{4}\right)+2 N$ \\
\hline$E_{6}$ & $3 A_{2}$ & $R\left(\pi_{1}\right) \otimes R\left(\pi_{1}\right) \otimes N+R\left(\pi_{2}\right) \otimes N \otimes R\left(\pi_{1}\right)+N \otimes R\left(\pi_{2}\right) \otimes R\left(\pi_{2}\right)$ \\
\hline$E_{6}$ & $A_{5}$ & $2 R\left(\pi_{1}\right)+R\left(\pi_{4}\right)$ \\
\hline$E_{6}$ & $D_{5}$ & $R\left(\pi_{1}\right)+R\left(\pi_{5}\right)+N$ \\
\hline$E_{8}$ & $2 A_{4}$ & $\mathrm{ad}+R\left(\pi_{1}\right) \otimes R\left(\pi_{3}\right)+R\left(\pi_{2}\right) \otimes R\left(\pi_{1}\right)+R\left(\pi_{3}\right) \otimes R\left(\pi_{4}\right)+R\left(\pi_{4}\right) \otimes R\left(\pi_{2}\right)$ \\
\hline$E_{8}$ & $2 D_{4}$ & $\mathrm{ad}+R\left(\pi_{1}\right) \otimes R\left(\pi_{1}\right)+R\left(\pi_{3}\right) \otimes R\left(\pi_{3}\right)+R\left(\pi_{4}\right) \otimes R\left(\pi_{4}\right)$ \\
\hline
\end{tabular}


Non-regular simple subalgebras of rank greater than 1 of the exceptional Lie algebras $\mathfrak{g}=E_{6}, E_{7}, E_{8}$

TABLE 6. $\mathfrak{g}=E_{6}$

\begin{tabular}{|c|c|c|c|c|c|}
\hline No. & $\mathfrak{h}$ & $\mathcal{R}(\mathfrak{h})^{L S}$ & $\Gamma(\mathfrak{h})^{L}$ & $n(\mathfrak{h})$ & $\Gamma(\mathfrak{h})$ \\
\hline \hline 1 & $\mathfrak{s l}_{3}$ & $E_{6}$ & $\mathbb{Z}_{2}$ & 2 & $\mathbb{Z}_{2}, \mathbb{Z}_{2}$ \\
\hline 2 & $G_{2}$ & $E_{6}$ & & 2 & \\
\hline 3 & $\mathfrak{s p}_{8}$ & $E_{6}$ & & 1 & \\
\hline 4 & $F_{4}$ & $E_{6}$ & & 1 & \\
\hline 5 & $\mathfrak{s l}_{3}$ & $2 A_{2}$ & $\mathbb{Z}_{2}$ & 1 & $\mathbb{Z}_{2}$ \\
\hline 6 & $\mathfrak{s l}_{3}$ & $2 A_{2}$ & 1 & 1 & 1 \\
\hline 7 & $\mathfrak{s l}_{3}$ & $A_{5}$ & 1 & 1 & 1 \\
\hline 8 & $\mathfrak{s o}_{5}$ & $A_{3}$ & & 1 & \\
\hline 9 & $\mathfrak{s o}_{5}$ & $A_{4}$ & & 1 & \\
\hline 10 & $G_{2}$ & $D_{4}$ & & 1 & \\
\hline 11 & $\mathfrak{s l}_{4}$ & $A_{5}$ & $\mathbb{Z}_{2}$ & 1 & $\mathbb{Z}_{2}$ \\
\hline 12 & $\mathfrak{s p}_{6}$ & $A_{5}$ & & 1 & \\
\hline 13 & $\mathfrak{s o}_{9}$ & $D_{5}$ & & 1 & \\
\hline 14 & $\mathfrak{s o}_{7}$ & $D_{4}$ & & 1 & \\
\hline 15 & $\mathfrak{s o}_{5}$ & $D_{5}$ & & 2 & \\
\hline 16 & $\mathfrak{s l}_{3}$ & $D_{4}, 3 A_{2}$ & $\mathbb{Z}_{2}$ & 1 & $\mathbb{Z}_{2}$ \\
\hline 17 & $\mathfrak{s l}_{3}$ & $3 A_{2}$ & $\mathbb{Z}_{2}$ & 1 & $\mathbb{Z}_{2}$ \\
\hline
\end{tabular}

TABLE 7. $\mathfrak{g}=E_{7}$

\begin{tabular}{|c|c|c|c|c|c|}
\hline No. & $\mathfrak{h}$ & $\mathcal{R}(\mathfrak{h})^{L S}$ & $\Gamma(\mathfrak{h})^{L}$ & $n(\mathfrak{h})$ & $\Gamma(\mathfrak{h})$ \\
\hline \hline 1 & $\mathfrak{s l}_{3}$ & $E_{7}$ & $\mathbb{Z}_{2}$ & 1 & $\mathbb{Z}_{2}$ \\
\hline 2 & $\mathfrak{s l}_{3}$ & $2 A_{2}$ & $\mathbb{Z}_{2}$ & 1 & $\mathbb{Z}_{2}$ \\
\hline 3 & $\mathfrak{s l}_{3}$ & $2 A_{2}$ & $\mathbb{Z}_{2}$ & 1 & $\mathbb{Z}_{2}$ \\
\hline 4 & $\mathfrak{s l}_{3}$ & $D_{4}, 3 A_{2}$ & $\mathbb{Z}_{2}$ & 1 & $\mathbb{Z}_{2}$ \\
\hline 5 & $\mathfrak{s l}_{3}$ & $3 A_{2}$ & $\mathbb{Z}_{2}$ & 1 & $\mathbb{Z}_{2}$ \\
\hline 6 & $\mathfrak{s l}_{3}$ & $A_{5}^{\prime}$ & $\mathbb{Z}_{2}$ & 1 & $\mathbb{Z}_{2}$ \\
\hline 7 & $\mathfrak{s o}_{5}$ & $A_{3}$ & & 1 & \\
\hline 8 & $G_{2}$ & $D_{4}$ & & 1 & \\
\hline 9 & $\mathfrak{s o}_{7}$ & $D_{4}$ & & 1 & \\
\hline
\end{tabular}


A. N. MINCHENKO

TABle 7. $\mathfrak{g}=E_{7}$ (continued)

\begin{tabular}{|c|c|c|c|c|c|}
\hline No. & $\mathfrak{h}$ & $\mathcal{R}(\mathfrak{h})^{L S}$ & $\Gamma(\mathfrak{h})^{L}$ & $n(\mathfrak{h})$ & $\Gamma(\mathfrak{h})$ \\
\hline \hline 10 & $\mathfrak{s p}_{6}$ & $A_{5}^{\prime}$ & & 1 & \\
\hline 11 & $\mathfrak{s o}_{9}$ & $D_{5}$ & & 1 & \\
\hline 12 & $F_{4}$ & $E_{6}$ & & 1 & \\
\hline 13 & $\mathfrak{s l}_{3}$ & $E_{6}$ & $\mathbb{Z}_{2}$ & 1 & $\mathbb{Z}_{2}$ \\
\hline 14 & $\mathfrak{s o}_{5}$ & $D_{5}$ & & 1 & \\
\hline 15 & $G_{2}$ & $E_{6}$ & & 1 & \\
\hline 16 & $\mathfrak{s l}_{4}$ & $A_{5}^{\prime}, 2 A_{3}$ & $\mathbb{Z}_{2}$ & 1 & $\mathbb{Z}_{2}$ \\
\hline 17 & $\mathfrak{s p}_{8}$ & $E_{6}, A_{7}$ & & 1 & \\
\hline 18 & $\mathfrak{s o}_{5}$ & $A_{4}, 2 A_{3}$ & & 1 & \\
\hline 19 & $\mathfrak{s l}_{3}$ & $A_{5}^{\prime \prime}$ & $\mathbb{Z}_{2}$ & 1 & $\mathbb{Z}_{2}$ \\
\hline 20 & $G_{2}$ & $A_{6}$ & & 1 & \\
\hline 21 & $\mathfrak{s p}_{6}$ & $A_{5}^{\prime \prime}$ & & 1 & \\
\hline 22 & $\mathfrak{s o}_{8}$ & $A_{7}$ & $\mathbb{S}_{3}$ & 1 & $\mathbb{S}_{3}$ \\
\hline 23 & $\mathfrak{s o}_{11}$ & $D_{6}$ & & 1 & \\
\hline 24 & $\mathfrak{s l}_{4}$ & $A_{5}^{\prime \prime}, 2 A_{3}$ & $\mathbb{Z}_{2}$ & 1 & $\mathbb{Z}_{2}$ \\
\hline 25 & $\mathfrak{s o}_{7}$ & $A_{6}, A_{7}$ & & 1 & \\
\hline 26 & $\mathfrak{s l}_{3}$ & $A_{7}, A_{5}+A_{2}$ & $\mathbb{Z}_{2}$ & 1 & $\mathbb{Z}_{2}$ \\
\hline 27 & $\mathfrak{s l}_{3}$ & $A_{5}+A_{2}$ & $\mathbb{Z}_{2}$ & 1 & $\mathbb{Z}_{2}$ \\
\hline
\end{tabular}

TABLE 8. $\mathfrak{g}=E_{8}$

\begin{tabular}{|c|c|c|c|c|c|}
\hline No. & $\mathfrak{h}$ & $\mathcal{R}(\mathfrak{h})^{L S}$ & $\Gamma(\mathfrak{h})^{L}$ & $n(\mathfrak{h})$ & $\Gamma(\mathfrak{h})$ \\
\hline \hline 1 & $\mathfrak{s o}_{5}$ & $E_{8}$ & & 1 & \\
\hline 2 & $\mathfrak{s l}_{3}$ & $D_{4}, 3 A_{2}$ & $\mathbb{Z}_{2}$ & 1 & $\mathbb{Z}_{2}$ \\
\hline 3 & $\mathfrak{s l}_{3}$ & $3 A_{2}$ & $\mathbb{Z}_{2}$ & 1 & $\mathbb{Z}_{2}$ \\
\hline 4 & $\mathfrak{s l}_{3}$ & $A_{5}$ & $\mathbb{Z}_{2}$ & 1 & $\mathbb{Z}_{2}$ \\
\hline 5 & $\mathfrak{s l}_{3}$ & $E_{6}$ & $\mathbb{Z}_{2}$ & 1 & $\mathbb{Z}_{2}$ \\
\hline 6 & $\mathfrak{s o}_{5}$ & $A_{3}$ & & 1 & \\
\hline 7 & $\mathfrak{s o}_{5}$ & $D_{5}$ & & 1 & \\
\hline 8 & $G_{2}$ & $D_{4}$ & & 1 & \\
\hline 9 & $G_{2}$ & $E_{6}$ & & 1 & \\
\hline 10 & $\mathfrak{s o}_{7}$ & $D_{4}$ & & 1 & \\
\hline
\end{tabular}


TABLE 8. $\mathfrak{g}=E_{8}$ (continued)

\begin{tabular}{|c|c|c|c|c|c|}
\hline No. & $\mathfrak{h}$ & $\mathcal{R}(\mathfrak{h})^{L S}$ & $\Gamma(\mathfrak{h})^{L}$ & $n(\mathfrak{h})$ & $\Gamma(\mathfrak{h})$ \\
\hline 11 & $\mathfrak{s p}_{6}$ & $A_{5}$ & & 1 & \\
\hline 12 & $\mathfrak{s o}_{9}$ & $D_{5}$ & & 1 & \\
\hline 13 & $F_{4}$ & $E_{6}$ & & 1 & \\
\hline 14 & $\mathfrak{s o}_{5}$ & $A_{4},\left(2 A_{3}\right)^{\prime \prime}$ & & 1 & \\
\hline 15 & $\mathfrak{s l}_{4}$ & $A_{5},\left(2 A_{3}\right)^{\prime \prime}$ & $\mathbb{Z}_{2}$ & 1 & $\mathbb{Z}_{2}$ \\
\hline 16 & $\mathfrak{s p}_{8}$ & $E_{6}, A_{7}^{\prime \prime}$ & & 1 & \\
\hline 17 & $\mathfrak{s l}_{3}$ & $2 A_{2}$ & $\mathbb{Z}_{2}$ & 1 & $\mathbb{Z}_{2}$ \\
\hline 18 & $\mathfrak{s l}_{3}$ & $A_{5}+A_{2}$ & $\mathbb{Z}_{2}$ & 1 & $\mathbb{Z}_{2}$ \\
\hline 19 & $\mathfrak{s l}_{3}$ & $E_{7}$ & $\mathbb{Z}_{2}$ & 1 & $\mathbb{Z}_{2}$ \\
\hline 20 & $\mathfrak{s o}_{11}$ & $D_{6}$ & & 1 & \\
\hline 21 & $G_{2}$ & $A_{6}, 2 D_{4}$ & & 1 & \\
\hline 22 & $\mathfrak{s o}_{7}$ & $A_{6}, A_{7}^{\prime \prime}, 2 D_{4}$ & & 1 & \\
\hline 23 & $\mathfrak{s l}_{3}$ & $A_{5}+A_{2}, A_{7}^{\prime}, A_{7}^{\prime \prime}, 2 D_{4}$ & $\mathbb{Z}_{2}$ & 2 & $\mathbb{Z}_{2}, \mathbb{Z}_{2}$ \\
\hline 24 & $\mathfrak{s o}_{8}$ & $A_{7}^{\prime \prime}, 2 D_{4}$ & $\mathbb{S}_{3}$ & 1 & $\mathbb{S}_{3}$ \\
\hline 25 & $\mathfrak{s o}_{8}$ & $2 D_{4}$ & $\mathbb{S}_{3}$ & 1 & $\mathbb{S}_{3}$ \\
\hline 26 & $\mathfrak{s o}_{8}$ & $A_{7}^{\prime}, 2 D_{4}$ & $\mathbb{Z}_{2}$ & 1 & $\mathbb{Z}_{2}$ \\
\hline 27 & $\mathfrak{s o}_{5}$ & $\left(2 A_{3}\right)^{\prime}$ & & 1 & \\
\hline 28 & $\mathfrak{s o}_{5}$ & $A_{3}+A_{4}$ & & 1 & \\
\hline 29 & $\mathfrak{s o}_{5}$ & $D_{7}$ & & 1 & \\
\hline 30 & $G_{2}$ & $D_{7}$ & & 1 & \\
\hline 31 & $\mathfrak{s l}_{4}$ & $D_{8}$ & $\mathbb{Z}_{2}$ & 1 & $\mathbb{Z}_{2}$ \\
\hline 32 & $\mathfrak{s p}_{6}$ & $D_{7}$ & & 1 & \\
\hline 33 & $\mathfrak{s p}_{8}$ & $A_{7}^{\prime}$ & & 1 & \\
\hline 34 & $\mathfrak{s o}_{13}$ & $D_{7}$ & & 1 & \\
\hline 35 & $\mathfrak{s o}_{15}$ & $D_{8}$ & & 1 & \\
\hline 36 & $\mathfrak{s o}_{7}$ & $A_{7}^{\prime}, 2 D_{4}$ & & 1 & \\
\hline 37 & $\mathfrak{s l}_{3}$ & $D_{4}+A_{2}, 4 A_{2}$ & $\mathbb{Z}_{2}$ & 1 & $\mathbb{Z}_{2}$ \\
\hline 38 & $\mathfrak{s l}_{4}$ & $\left(2 A_{3}\right)^{\prime}$ & $\mathbb{Z}_{2}$ & 1 & $\mathbb{Z}_{2}$ \\
\hline 39 & $\mathfrak{s l}_{3}$ & $E_{6}+A_{2}$ & $\mathbb{Z}_{2}$ & 1 & 1 \\
\hline 40 & $\mathfrak{s l}_{5}$ & $2 A_{4}$ & $\mathbb{Z}_{2}$ & 1 & $\mathbb{Z}_{2}$ \\
\hline 41 & $\mathfrak{s o}_{5}$ & $2 A_{4}, A_{3}+D_{5}$ & & 1 & \\
\hline 42 & $\mathfrak{s o}_{9}$ & $A_{8}, D_{8}$ & & 1 & \\
\hline 43 & $\mathfrak{s o}_{9}$ & $D_{8}$ & & 1 & \\
\hline
\end{tabular}


TABLE 9. Cases of partition of the equivalence classes of embeddings into exceptional Lie algebras

\begin{tabular}{|c|c|c|}
\hline $\mathfrak{g}$ & $\mathfrak{h}$ & $\varphi^{L}$ \\
\hline \hline$E_{6}$ & $\mathfrak{s l}_{3}$ & $\varphi_{1}: \mathfrak{h} \hookrightarrow E_{6}, \quad \varphi_{2}=\sigma \circ \varphi_{1}$ \\
\hline & $G_{2}$ & $\varphi_{1}: \mathfrak{h} \hookrightarrow E_{6}, \quad \varphi_{2}=\sigma \circ \varphi_{1}$ \\
\hline & $\mathfrak{s o}_{5}$ & $\varphi_{1}: \mathfrak{h} \hookrightarrow D_{5}, \quad \varphi_{2}=\sigma \circ \varphi_{1}$ \\
\hline$E_{7}$ & $\mathfrak{s l}_{3}+\mathfrak{s l}_{2}+\mathfrak{s l}_{2}\left(+\mathfrak{s l}_{2}\right)$ & $\varphi_{1}: \mathfrak{h} \hookrightarrow D_{4}+A_{1}+A_{1}\left(+A_{1}\right), \quad \varphi_{2}=\sigma \circ \varphi_{1}$ \\
\hline$E_{8}$ & $\mathfrak{h}_{0}+\mathfrak{s l}_{3}$ & $\varphi_{1}: \mathfrak{h} \hookrightarrow E_{6}+A_{2}, \quad \varphi_{2}=\varphi_{1} \circ(\operatorname{Id} \times \tau)$, \\
& & $\mathfrak{h}_{0}=\mathfrak{s l}_{3}, \mathfrak{s o}_{5}, G_{2}$ \\
\hline & $\mathfrak{h}_{0}+\mathfrak{s l}_{3}$ & $\varphi_{1}: \mathfrak{h} \hookrightarrow D_{4}+D_{4}, \quad \varphi_{2}=\varphi_{1} \circ(\operatorname{Id} \times \tau)$, \\
& & $\mathfrak{h}=\mathfrak{s o}_{8}, \mathfrak{s l}_{3}, \mathfrak{s l}_{2}+\mathfrak{s l}_{2}+\mathfrak{s l}_{2}\left(+\mathfrak{s l}_{2}\right)$ \\
\hline & $\mathfrak{s o}_{5}+\mathfrak{s l}_{4}$ & $\varphi_{1}: \mathfrak{h} \hookrightarrow D_{5}+A_{3}, \quad \varphi_{2}=\varphi_{1} \circ(\operatorname{Id} \times \tau)$ \\
\hline & $\mathfrak{s l}_{3}$ & $\varphi_{1}: \mathfrak{h} \hookrightarrow E_{6}+A_{2}, \quad \varphi_{2}=\varphi_{1} \circ \tau$ \\
\hline & $\mathfrak{s l}_{3}$ & $\varphi_{1}: \mathfrak{h} \hookrightarrow D_{4}+D_{4}, \quad \varphi_{2}=(\sigma \times \operatorname{Id}) \varphi_{1}$ \\
\hline
\end{tabular}

The centralizers of simple subalgebras of rank greater than 1 in exceptional Lie groups

TABLE $10 . G=G_{2}$

\begin{tabular}{|c|c|c|c|c|c|}
\hline $\mathfrak{h}$ & $\mathcal{R}(\mathfrak{h})^{S}$ & $\mathcal{D}(\mathfrak{h})$ & $\mathfrak{z}$ & $Z$ & $N$ \\
\hline \hline$A_{2}^{1}$ & $A_{2}$ & $\mathbb{Z}_{3}$ & 0 & $\mathbb{Z}_{3}$ & $\mathbb{S}_{3}$ \\
\hline
\end{tabular}

TABLE $11 . G=F_{4}$

\begin{tabular}{|c|c|c|c|c|c|}
\hline $\mathfrak{h}$ & $\mathcal{R}(\mathfrak{h})^{S}$ & $\mathcal{D}(\mathfrak{h})$ & $\mathfrak{z}$ & $Z$ & $N$ \\
\hline \hline$A_{2}^{1}$ & $A_{2}$ & $\mathrm{~T}_{2}$ & $A_{2}^{2}$ & $\mathrm{SL}_{3}$ & $\mathbb{Z}_{2} \curlywedge Z$ \\
\hline$A_{2}^{2}$ & $\widetilde{A_{2}}$ & $\mathrm{~T}_{2}$ & $A_{2}^{1}$ & $\mathrm{SL}_{3}$ & $\mathbb{Z}_{2} \curlywedge Z$ \\
\hline$A_{2}^{3^{\prime}}$ & $D_{4} ; A_{2}+\widetilde{A_{2}}$ & $\mathbb{V}_{4} ; \mathbb{Z}_{3}$ & 0 & $\mathbb{A}_{4}$ & $\mathbb{S}_{4}$ \\
\hline$A_{2}^{3^{\prime \prime}}$ & $A_{2}+\widetilde{A_{2}}$ & $\mathbb{Z}_{3}$ & 0 & $\mathbb{Z}_{3}$ & $\mathbb{S}_{3}$ \\
\hline$B_{2}^{1}$ & $B_{2} ; A_{3}$ & $\mathrm{~T}_{2} ; \mathbb{Z}_{2} \times \mathrm{T}_{1}$ & $2 A_{1}^{1}$ & $\mathbb{Z}_{2} \wedge\left(\mathrm{SL}_{2}{ }^{2}\right)$ & $Z$ \\
\hline$G_{2}^{1}$ & $B_{3} ; D_{4}$ & $\mathrm{~T}_{1} ; \mathbb{V}_{4}$ & $A_{1}^{8}$ & $\mathrm{SO}_{3}$ & $Z$ \\
\hline$A_{3}^{1}$ & $A_{3}$ & $\mathbb{Z}_{2} \times \mathrm{T}_{1}$ & $A_{1}^{2}$ & $\mathbb{Z}_{2} \times \mathrm{SL}_{2}$ & $\mathbb{Z}_{2} \curlywedge Z$ \\
\hline$B_{3}^{1}$ & $B_{3} ; D_{4}$ & $\mathrm{~T}_{1} ; \mathbb{V}_{4}$ & $T_{1}$ & $\mathbb{Z}_{2} \wedge \mathrm{T}_{1}$ & $Z$ \\
\hline
\end{tabular}


TABle $11 . G=F_{4}$ (continued)

\begin{tabular}{|c|c|c|c|c|c|}
\hline $\mathfrak{h}$ & $\mathcal{R}(\mathfrak{h})^{S}$ & $\mathcal{D}(\mathfrak{h})$ & $\mathfrak{z}$ & $Z$ & $N$ \\
\hline \hline$C_{3}^{1}$ & $C_{3}$ & $\mathrm{~T}_{1}$ & $A_{1}^{1}$ & $\mathrm{SL}_{2}$ & $Z$ \\
\hline$B_{4}^{1}$ & $B_{4}$ & $\mathbb{Z}_{2}$ & 0 & $\mathbb{Z}_{2}$ & $Z$ \\
\hline$D_{4}^{1}$ & $D_{4}$ & $\mathbb{V}_{4}$ & 0 & $\mathbb{V}_{4}$ & $\mathbb{S}_{4}$ \\
\hline
\end{tabular}

TABLE $12 . G=E_{6}$

\begin{tabular}{|c|c|c|c|c|c|}
\hline $\mathfrak{h}$ & $\mathcal{R}(\mathfrak{h})^{S}$ & $\mathcal{D}(\mathfrak{h})$ & $\mathfrak{z}$ & $Z$ & $N$ \\
\hline$A_{2}^{1}$ & $A_{2}$ & $\mathrm{~T}_{4}$ & $2 A_{2}^{1}$ & $\left(\mathrm{SL}_{3}{ }^{2}\right) / \mathbb{Z}_{3}$ & $\mathbb{Z}_{2} \wedge Z$ \\
\hline$A_{2}^{2^{\prime}}$ & $2 A_{2}$ & $\mathrm{~T}_{2}$ & $A_{2}^{1}$ & $\mathrm{SL}_{3}$ & $\mathbb{Z}_{2} \curlywedge Z$ \\
\hline$A_{2}^{2^{\prime \prime}}$ & $2 A_{2}$ & $\mathrm{~T}_{2}$ & $G_{2}^{1}$ & $G_{2}$ & $Z$ \\
\hline$A_{2}^{3^{\prime}}$ & $D_{4} ; 3 A_{2}$ & $\mathrm{~T}_{2} ; \mathbb{Z}_{3}$ & $T_{2}$ & $\mathbb{Z}_{3}<\mathrm{T}_{2}$ & $\mathbb{S}_{3} \wedge Z^{\circ}$ \\
\hline$A_{2}^{3^{\prime \prime}}$ & $3 A_{2}$ & $\mathbb{Z}_{3}$ & 0 & $\mathbb{Z}_{3}$ & $\mathbb{S}_{3}$ \\
\hline$A_{2}^{5}$ & $A_{5}$ & $\mathrm{~T}_{1}$ & $A_{1}^{1}$ & $\mathrm{SL}_{2}$ & $Z$ \\
\hline$A_{2}^{9}(1)$ & $E_{6}$ & 1 & 0 & 1 & $\mathbb{Z}_{2}$ \\
\hline$A_{2}^{9}(2)$ & $E_{6}$ & 1 & 0 & 1 & $\mathbb{Z}_{2}$ \\
\hline$B_{2}^{1}$ & $A_{3}$ & $\mathrm{~T}_{3}$ & $B_{2}^{1}+T_{1}$ & $\mathrm{Sp}_{4} \cdot \mathrm{T}_{1}$ & $Z$ \\
\hline$B_{2}^{2}$ & $A_{4}$ & $\mathrm{~T}_{2}$ & $A_{1}^{1}+T_{1}$ & $\mathrm{SL}_{2} \cdot \mathrm{T}_{1}$ & $Z$ \\
\hline$B_{2}^{3}(1)$ & $D_{5}$ & $\mathrm{~T}_{1}$ & $T_{1}$ & $\mathrm{~T}_{1}$ & $Z$ \\
\hline$B_{2}^{3}(2)$ & $D_{5}$ & $\mathrm{~T}_{1}$ & $T_{1}$ & $\mathrm{~T}_{1}$ & $Z$ \\
\hline$G_{2}^{1}$ & $D_{4}$ & $\mathrm{~T}_{2}$ & $A_{2}^{2^{\prime \prime}}$ & $\mathrm{PSL}_{3}$ & $Z$ \\
\hline$G_{2}^{3}(1)$ & $E_{6}$ & 1 & 0 & 1 & $Z$ \\
\hline$G_{2}^{3}(2)$ & $E_{6}$ & 1 & 0 & 1 & $Z$ \\
\hline$A_{3}^{1}$ & $A_{3}$ & $\mathrm{~T}_{3}$ & $2 A_{1}^{1}+T_{1}$ & $\left(\mathrm{SL}_{2}^{2}\right) \cdot \mathrm{T}_{1}$ & $\mathbb{Z}_{2} \wedge Z$ \\
\hline$A_{3}^{2}$ & $A_{5}$ & $\mathrm{~T}_{1}$ & $A_{1}^{1}$ & $\mathrm{SL}_{2}$ & $\mathbb{Z}_{2} \times Z$ \\
\hline$B_{3}^{1}$ & $D_{4}$ & $\mathrm{~T}_{2}$ & $A_{1}^{2}+T_{1}$ & $\mathrm{SL}_{2} \cdot \mathrm{T}_{1}$ & $Z$ \\
\hline$C_{3}^{1}$ & $A_{5}$ & $\mathrm{~T}_{1}$ & $A_{1}^{1}$ & $\mathrm{SL}_{2}$ & $Z$ \\
\hline$A_{4}^{1}$ & $A_{4}$ & $\mathrm{~T}_{2}$ & $A_{1}^{1}+T_{1}$ & $\mathrm{SL}_{2} \cdot \mathrm{T}_{1}$ & $Z$ \\
\hline$B_{4}^{1}$ & $D_{5}$ & $\mathrm{~T}_{1}$ & $T_{1}$ & $\mathrm{~T}_{1}$ & $Z$ \\
\hline$C_{4}^{1}$ & $E_{6}$ & 1 & 0 & 1 & $Z$ \\
\hline$D_{4}^{1}$ & $D_{4}$ & $\mathrm{~T}_{2}$ & $T_{2}$ & $\mathrm{~T}_{2}$ & $\mathbb{S}_{3} \wedge Z$ \\
\hline$F_{4}^{1}$ & $E_{6}$ & 1 & 0 & 1 & $Z$ \\
\hline$A_{5}^{1}$ & $A_{5}$ & $\mathrm{~T}_{1}$ & $A_{1}^{1}$ & $\mathrm{SL}_{2}$ & $Z$ \\
\hline$D_{5}^{1}$ & $D_{5}$ & $\mathrm{~T}_{1}$ & $T_{1}$ & $\mathrm{~T}_{1}$ & $Z$ \\
\hline
\end{tabular}


A. N. MINCHENKO

TABLE $13 . G=E_{7}$

\begin{tabular}{|c|c|c|c|c|c|}
\hline $\mathfrak{h}$ & $\mathcal{R}(\mathfrak{h})^{S}$ & $\mathcal{D}(\mathfrak{h})$ & $\mathfrak{z}$ & $Z$ & $N$ \\
\hline$A_{2}^{1}$ & $A_{2}$ & $\mathrm{~T}_{5}$ & $A_{5}^{\prime \prime}$ & $\mathrm{SL}_{6} / \mathbb{Z}_{2}$ & $\mathbb{Z}_{2} \wedge Z$ \\
\hline$A_{2}^{2^{\prime}}$ & $2 A_{2}$ & $\mathrm{~T}_{3}$ & $A_{2}^{1}+A_{1}^{3^{\prime \prime}}$ & $\mathrm{SL}_{3} \times \mathrm{SO}_{3}$ & $\mathbb{Z}_{2} \wedge Z$ \\
\hline$A_{2}^{2^{\prime \prime}}$ & $2 A_{2}$ & $\mathrm{~T}_{3}$ & $G_{2}^{1}+T_{1}$ & $G_{2} \times \mathrm{T}_{1}$ & $\mathbb{Z}_{2} \wedge Z$ \\
\hline$A_{2}^{3^{\prime}}$ & $D_{4} ; 3 A_{2}$ & $\mathrm{~T}_{3} ; \mathbb{Z}_{3} \times \mathrm{T}_{1}$ & $3 A_{1}^{1}$ & $\mathbb{Z}_{3} \curlywedge\left(\left(\mathrm{SL}_{2}{ }^{3}\right) / \mathbb{Z}_{2}\right)$ & $\mathbb{S}_{3} \measuredangle Z^{\circ}$ \\
\hline$A_{2}^{3^{\prime \prime}}$ & $3 A_{2}$ & $\mathbb{Z}_{3} \times \mathrm{T}_{1}$ & $A_{1}^{3^{\prime \prime}}$ & $\mathbb{Z}_{3} \times \mathrm{SO}_{3}$ & $\mathbb{S}_{3} \times Z^{\circ}$ \\
\hline$A_{2}^{5^{\prime}}$ & $A_{5}^{\prime}$ & $\mathrm{T}_{2}$ & $A_{1}^{1}+T_{1}$ & $\mathrm{SL}_{2} \times \mathrm{T}_{1}$ & $\mathbb{Z}_{2} \wedge Z$ \\
\hline$A_{2}^{5^{\prime \prime}}$ & $A_{5}^{\prime \prime}$ & $\mathrm{T}_{2}$ & $A_{2}^{1}$ & $\mathrm{SL}_{3}$ & $\mathbb{Z}_{2} \wedge Z$ \\
\hline$A_{2}^{6^{\prime}}$ & $A_{5}+A_{2} ; A_{7}$ & $\mathbb{Z}_{3} ; \mathbb{Z}_{2}$ & 0 & $\mathbb{A}_{4}$ & $\mathbb{S}_{4}$ \\
\hline$A_{2}^{6^{\prime \prime}}$ & $A_{5}+A_{2}$ & $\mathbb{Z}_{3}$ & 0 & $\mathbb{Z}_{3}$ & $\mathbb{S}_{3}$ \\
\hline$A_{2}^{9}$ & $E_{6}$ & $\mathrm{~T}_{1}$ & $T_{1}$ & $\mathrm{~T}_{1}$ & $\mathbb{Z}_{2} \times Z$ \\
\hline$A_{2}^{21}$ & $E_{7}$ & 1 & 0 & 1 & $\mathbb{Z}_{2}$ \\
\hline$B_{2}^{1}$ & $A_{3}$ & $\mathrm{~T}_{4}$ & $B_{3}^{1}+A_{1}^{1}$ & $\left(\operatorname{Spin}_{7} \times \mathrm{SL}_{2}\right) / \mathbb{Z}_{2}$ & $Z$ \\
\hline$B_{2}^{2}$ & $A_{4} ; 2 A_{3}$ & $\mathrm{~T}_{3} ; \mathbb{Z}_{2} \times \mathrm{T}_{1}$ & $A_{2}^{1}+T_{1}$ & $\mathbb{Z}_{2} \curlywedge\left(\mathrm{SL}_{3} \cdot \mathrm{T}_{1}\right)$ & $Z$ \\
\hline$B_{2}^{3}$ & $D_{5}$ & $\mathrm{~T}_{2}$ & $A_{1}^{1}+T_{1}$ & $\mathrm{SL}_{2} \cdot \mathrm{T}_{1}$ & $Z$ \\
\hline$G_{2}^{1}$ & $D_{4}$ & $\mathrm{~T}_{3}$ & $C_{3}^{1^{\prime \prime}}$ & $\mathrm{PSp}_{6}$ & $Z$ \\
\hline$G_{2}^{2}$ & $A_{6}$ & $\mathrm{~T}_{1}$ & $A_{1}^{7}$ & $\mathrm{SO}_{3}$ & $Z$ \\
\hline$G_{2}^{3}$ & $E_{6}$ & $\mathrm{~T}_{1}$ & $T_{1}$ & $\mathrm{~T}_{1}$ & $Z$ \\
\hline$A_{3}^{1}$ & $A_{3}$ & $\mathrm{~T}_{4}$ & $A_{3}^{1}+A_{1}^{1}$ & $\left(\mathrm{SL}_{4} \times \mathrm{SL}_{2}\right) / \mathbb{Z}_{2}$ & $\mathbb{Z}_{2} \wedge Z$ \\
\hline$A_{3}^{2^{\prime}}$ & $A_{5}^{\prime} ; 2 A_{3}$ & $\mathrm{~T}_{2} ; \mathbb{Z}_{2} \times \mathrm{T}_{1}$ & $A_{1}^{1}+T_{1}$ & $\mathbb{Z}_{2} \curlywedge\left(\mathrm{SL}_{2} \times \mathrm{T}_{1}\right)$ & $\mathbb{Z}_{2} \times Z$ \\
\hline$A_{3}^{2^{\prime \prime}}$ & $A_{5}^{\prime \prime} ; 2 A_{3}$ & $\mathrm{~T}_{2} ; \mathbb{Z}_{2} \times \mathrm{T}_{1}$ & $A_{2}^{1}$ & $\mathbb{Z}_{2} \wedge \mathrm{SL}_{3}$ & $\mathbb{Z}_{2} \times Z$ \\
\hline$B_{3}^{1}$ & $D_{4}$ & $\mathrm{~T}_{3}$ & $B_{2}^{1}+A_{1}^{1}$ & $\left(\mathrm{Sp}_{4} \times \mathrm{SL}_{2}\right) / \mathbb{Z}_{2}$ & $Z$ \\
\hline$B_{3}^{2}$ & $A_{6} ; A_{7}$ & $\mathrm{~T}_{1} ; \mathbb{Z}_{2}$ & $T_{1}$ & $\mathbb{Z}_{2} \nprec \mathrm{T}_{1}$ & $Z$ \\
\hline$C_{3}^{1^{\prime}}$ & $A_{5}^{\prime}$ & $\mathrm{T}_{2}$ & $A_{1}^{1}+A_{1}^{3^{\prime \prime}}$ & $\mathrm{SL}_{2} \times \mathrm{SO}_{3}$ & $Z$ \\
\hline$C_{3}^{1^{\prime \prime}}$ & $A_{5}^{\prime \prime}$ & $\mathrm{T}_{2}$ & $G_{2}^{1}$ & $G_{2}$ & $Z$ \\
\hline$A_{4}^{1}$ & $A_{4}$ & $\mathrm{~T}_{3}$ & $A_{2}^{1}+T_{1}$ & $\mathrm{SL}_{3} \cdot \mathrm{T}_{1}$ & $\mathbb{Z}_{2} \curlywedge Z$ \\
\hline$B_{4}^{1}$ & $D_{5}$ & $\mathrm{~T}_{2}$ & $A_{1}^{1}+A_{1}^{2}$ & $\left(\mathrm{SL}_{2}^{2}\right) / \mathbb{Z}_{2}$ & $Z$ \\
\hline$C_{4}^{1}$ & $E_{6} ; A_{7}$ & $\mathrm{~T}_{1} ; \mathbb{Z}_{2}$ & $T_{1}$ & $\mathbb{Z}_{2} \curlywedge \mathrm{T}_{1}$ & $Z$ \\
\hline$D_{4}^{1}$ & $D_{4}$ & $\mathrm{~T}_{3}$ & $3 A_{1}^{1}$ & $\left(\mathrm{SL}_{2}{ }^{3}\right) / \mathbb{Z}_{2}$ & $\mathbb{S}_{3} \wedge Z$ \\
\hline$D_{4}^{2}$ & $A_{7}$ & $\mathbb{Z}_{2}$ & 0 & $\mathbb{V}_{4}$ & $\mathbb{S}_{4}$ \\
\hline$F_{4}^{1}$ & $E_{6}$ & $\mathrm{~T}_{1}$ & $A_{1}^{3^{\prime \prime}}$ & $\mathrm{SO}_{3}$ & $Z$ \\
\hline$A_{5}^{1^{\prime}}$ & $A_{5}^{\prime}$ & $\mathrm{T}_{2}$ & $A_{1}^{1}+T_{1}$ & $\mathrm{SL}_{2} \times \mathrm{T}_{1}$ & $\mathbb{Z}_{2} \wedge Z$ \\
\hline
\end{tabular}


TABle 13. $G=E_{7}$ (continued)

\begin{tabular}{|c|c|c|c|c|c|}
\hline $\mathfrak{h}$ & $\mathcal{R}(\mathfrak{h})^{S}$ & $\mathcal{D}(\mathfrak{h})$ & $\mathfrak{z}$ & $Z$ & $N$ \\
\hline \hline$A_{5}^{1 \prime \prime}$ & $A_{5}^{\prime \prime}$ & $\mathrm{T}_{2}$ & $A_{2}^{1}$ & $\mathrm{SL}_{3}$ & $\mathbb{Z}_{2} \wedge Z$ \\
\hline$B_{5}^{1}$ & $D_{6}$ & $\mathrm{~T}_{1}$ & $A_{1}^{1}$ & $\mathrm{SL}_{2}$ & $Z$ \\
\hline$D_{5}^{1}$ & $D_{5}$ & $\mathrm{~T}_{2}$ & $A_{1}^{1}+T_{1}$ & $\mathrm{SL}_{2} \cdot \mathrm{T}_{1}$ & $\mathbb{Z}_{2} \curlywedge Z$ \\
\hline$A_{6}^{1}$ & $A_{6}$ & $\mathrm{~T}_{1}$ & $T_{1}$ & $\mathrm{~T}_{1}$ & $\mathbb{Z}_{2} \curlywedge Z$ \\
\hline$D_{6}^{1}$ & $D_{6}$ & $\mathrm{~T}_{1}$ & $A_{1}^{1}$ & $\mathrm{SL}_{2}$ & $Z$ \\
\hline$E_{6}^{1}$ & $E_{6}$ & $\mathrm{~T}_{1}$ & $T_{1}$ & $\mathrm{~T}_{1}$ & $\mathbb{Z}_{2} \curlywedge Z$ \\
\hline$A_{7}^{1}$ & $A_{7}$ & $\mathbb{Z}_{2}$ & 0 & $\mathbb{Z}_{2}$ & $\mathbb{V}_{4}$ \\
\hline
\end{tabular}

TABle $14 . G=E_{8}$

\begin{tabular}{|c|c|c|c|c|c|}
\hline $\mathfrak{h}$ & $\mathcal{R}(\mathfrak{h})^{S}$ & $\mathcal{D}(\mathfrak{h})$ & $\mathfrak{z}$ & $Z$ & $N$ \\
\hline \hline$A_{2}^{1}$ & $A_{2}$ & $\mathrm{~T}_{6}$ & $E_{6}^{1}$ & $\widehat{E}_{6}$ & $\mathbb{Z}_{2} \wedge Z$ \\
\hline$A_{2}^{2}$ & $2 A_{2}$ & $\mathrm{~T}_{4}$ & $G_{2}^{1}+A_{2}^{1}$ & $G_{2} \times \mathrm{SL}_{3}$ & $\mathbb{Z}_{2} \wedge Z$ \\
\hline$A_{2}^{3^{\prime}}$ & $D_{4} ; 3 A_{2}$ & $\mathrm{~T}_{4} ; \mathbb{Z}_{3} \times \mathrm{T}_{2}$ & $D_{4}^{1}$ & $\mathbb{Z}_{3} \wedge \mathrm{Spin}_{8}$ & $\mathbb{S}_{3} \wedge Z^{\circ}$ \\
\hline$A_{2}^{3^{\prime \prime}}$ & $3 A_{2}$ & $\mathbb{Z}_{3} \times \mathrm{T}_{2}$ & $G_{2}^{1}$ & $\mathbb{Z}_{3} \times G_{2}$ & $\mathbb{S}_{3} \times Z^{\circ}$ \\
\hline$A_{2}^{4}$ & $D_{4}+A_{2} ; 4 A_{2}$ & $\mathrm{~T}_{2} ; \mathbb{Z}_{3}^{3}$ & $T_{2}$ & $\mathbb{Z}_{3} \wedge \mathrm{T}_{2}$ & $\mathbb{S}_{3} \wedge Z^{\circ}$ \\
\hline$A_{2}^{5}$ & $A_{5}$ & $\mathrm{~T}_{3}$ & $A_{2}^{1}+A_{1}^{1}$ & $\mathrm{SL}_{3} \times \mathrm{SL}_{2}$ & $\mathbb{Z}_{2} \wedge Z$ \\
\hline$A_{2}^{6^{\prime}}(1)$ & $A_{5}+A_{2} ; A_{7}^{\prime \prime} ; 2 D_{4}$ & $\mathbb{Z}_{3} \times \mathrm{T}_{1} ; \mathbb{Z}_{2} \times \mathrm{T}_{1} ; \mathbb{V}_{4}$ & $A_{1}^{1}$ & $\mathbb{A}_{4} \times \mathrm{SL}_{2}$ & $\mathbb{S}_{4} \times Z^{\circ}$ \\
\hline$A_{2}^{6^{\prime}}(2)$ & $A_{7}^{\prime} ; 2 D_{4}$ & $\mathrm{~T}_{1} ; \mathbb{V}_{4}$ & $A_{1}^{16}$ & $\mathrm{SO}_{3}$ & $\mathbb{Z}_{2} \times Z$ \\
\hline$A_{2}^{6^{\prime \prime}}$ & $A_{5}+A_{2}$ & $\mathbb{Z}_{3} \times \mathrm{T}_{1}$ & $A_{1}^{1}$ & $\mathbb{Z}_{3} \times \mathrm{SL}_{2}$ & $\mathbb{S}_{3} \times Z^{\circ}$ \\
\hline$A_{2}^{9}$ & $E_{6}$ & $\mathrm{~T}_{2}$ & $A_{2}^{1}$ & $\mathrm{SL}_{3}$ & $\mathbb{Z}_{2} \times Z$ \\
\hline$A_{2}^{10}$ & $E_{6}+A_{2}$ & $\mathbb{Z}_{3}$ & 0 & $\mathbb{Z}_{3}$ & $Z$ \\
\hline$A_{2}^{21}$ & $E_{7}$ & $\mathrm{~T}_{1}$ & $A_{1}^{1}$ & $\mathrm{SL}_{2}$ & $\mathbb{Z}_{2} \times Z$ \\
\hline$B_{2}^{1}$ & $A_{3}$ & $\mathrm{~T}_{5}$ & $B_{5}^{1}$ & $\mathrm{Spin}_{11}$ & $Z$ \\
\hline$B_{2}^{2^{\prime}}$ & $A_{4} ;\left(2 A_{3}\right)^{\prime \prime}$ & $\mathrm{T}_{4} ; \mathbb{Z}_{2} \times \mathrm{T}_{2}$ & $A_{4}$ & $\mathbb{Z}_{2} \curlywedge \mathrm{SL}_{5}$ & $Z$ \\
\hline$B_{2}^{2^{\prime \prime}}$ & $\left(2 A_{3}\right)^{\prime}$ & $\mathrm{T}_{2}$ & $B_{2}^{2^{\prime \prime}}$ & $\mathrm{Sp}_{4}$ & $Z$ \\
\hline$B_{2}^{3^{\prime}}$ & $D_{5}$ & $\mathrm{~T}_{3}$ & $A_{3}$ & $\mathrm{SL}_{4}$ & $Z$ \\
\hline$B_{2}^{3^{\prime \prime}}$ & $A_{4}+A_{3}$ & $\mathrm{~T}_{1}$ & $A_{1}^{10^{\prime \prime}}$ & $\mathrm{SL}_{4}$ & $Z$ \\
\hline$B_{2}^{4}$ & $2 A_{4} ; D_{5}+A_{3}$ & $\mathbb{Z}_{5} ; \mathbb{Z}_{4}$ & 0 & $\mathbb{Z}_{4} \wedge \mathbb{Z}_{5}$ & $Z$ \\
\hline$B_{2}^{7}$ & $D_{7}$ & $\mathrm{~T}_{1}$ & $\mathrm{~T}_{1}$ & $Z$ \\
\hline$B_{2}^{12}$ & $E_{8}$ & 1 & 0 & $Z$ \\
\hline
\end{tabular}


A. N. MINCHENKO

TABle $14 . G=E_{8}$ (continued)

\begin{tabular}{|c|c|c|c|c|c|}
\hline $\mathfrak{h}$ & $\mathcal{R}(\mathfrak{h})^{S}$ & $\mathcal{D}(\mathfrak{h})$ & $\mathfrak{z}$ & $Z$ & $N$ \\
\hline$G_{2}^{1}$ & $D_{4}$ & $\mathrm{~T}_{4}$ & $F_{4}^{1}$ & $F_{4}$ & $Z$ \\
\hline$G_{2}^{2}$ & $A_{6} ; 2 D_{4}$ & $\mathrm{~T}_{2} ; \mathbb{V}_{4}$ & $A_{1}^{1}+A_{1}^{7}$ & $\mathrm{SL}_{2} \times \mathrm{SO}_{3}$ & $Z$ \\
\hline$G_{2}^{3}$ & $E_{6}$ & $\mathrm{~T}_{2}$ & $A_{2}^{1}$ & $\mathrm{SL}_{3}$ & $Z$ \\
\hline$G_{2}^{4}$ & $D_{7}$ & $\mathrm{~T}_{1}$ & $T_{1}$ & $\mathrm{~T}_{1}$ & $Z$ \\
\hline$A_{3}^{1}$ & $A_{3}$ & $\mathrm{~T}_{5}$ & $D_{5}^{1}$ & $\operatorname{Spin}_{10}$ & $\mathbb{Z}_{2} \wedge Z$ \\
\hline$A_{3}^{2^{\prime}}$ & $A_{5} ;\left(2 A_{3}\right)^{\prime \prime}$ & $\mathrm{T}_{3} ; \mathbb{Z}_{2} \times \mathrm{T}_{2}$ & $A_{2}^{1}+A_{1}^{1}$ & $\left(\mathbb{Z}_{2} \curlywedge \mathrm{SL}_{3}\right) \times \mathrm{SL}_{2}$ & $\mathbb{Z}_{2} \times Z$ \\
\hline$A_{3}^{2^{\prime \prime}}$ & $\left(2 A_{3}\right)^{\prime}$ & $\mathrm{T}_{2}$ & $A_{1}^{4^{\prime}}+T_{1}$ & $\mathrm{SL}_{2} \cdot \mathrm{T}_{1}$ & $\mathbb{Z}_{2} \wedge Z$ \\
\hline$A_{3}^{4}$ & $D_{8}$ & $\mathbb{Z}_{2}$ & 0 & $\mathbb{Z}_{2}$ & $\mathbb{V}_{4}$ \\
\hline$B_{3}^{1}$ & $D_{4}$ & $\mathrm{~T}_{4}$ & $B_{4}^{1}$ & $\operatorname{Spin}_{9}$ & $Z$ \\
\hline$B_{3}^{2^{\prime}}$ & $A_{6} ; A_{7}^{\prime \prime} ; 2 D_{4}$ & $\mathrm{~T}_{2} ; \mathbb{Z}_{2} \times \mathrm{T}_{1} ; \mathbb{V}_{4}$ & $A_{1}^{1}+T_{1}$ & $\left(\mathbb{Z}_{2}<\mathrm{T}_{1}\right) \times \mathrm{SL}_{2}$ & $Z$ \\
\hline$B_{3}^{2^{\prime \prime}}$ & $A_{7}^{\prime} ; 2 D_{4}$ & $\mathrm{~T}_{1} ; \mathbb{V}_{4}$ & $T_{1}$ & $\mathbb{Z}_{2} \curlywedge \mathrm{T}_{1}$ & $Z$ \\
\hline$C_{3}^{1}$ & $A_{5}$ & $\mathrm{~T}_{3}$ & $G_{2}^{1}+A_{1}^{1}$ & $G_{2} \times \mathrm{SL}_{2}$ & $Z$ \\
\hline$C_{3}^{2}$ & $D_{7}$ & $\mathrm{~T}_{1}$ & $T_{1}$ & $\mathrm{~T}_{1}$ & $Z$ \\
\hline$A_{4}^{1}$ & $A_{4}$ & $\mathrm{~T}_{4}$ & $A_{4}^{1}$ & $\mathrm{SL}_{5}$ & $\mathbb{Z}_{2} \wedge Z$ \\
\hline$A_{4}^{2}$ & $2 A_{4}$ & $\mathbb{Z}_{5}$ & 0 & $\mathbb{Z}_{5}$ & $\mathbb{Z}_{2} \wedge Z$ \\
\hline$B_{4}^{1}$ & $D_{5}$ & $\mathrm{~T}_{3}$ & $B_{3}^{1}$ & $\operatorname{Spin}_{7}$ & $Z$ \\
\hline$B_{4}^{2^{\prime}}$ & $A_{8} ; D_{8}$ & $\mathbb{Z}_{3} ; \mathbb{Z}_{2}$ & 0 & $\mathbb{S}_{3}$ & $Z$ \\
\hline$B_{4}^{2^{\prime \prime}}$ & $D_{8}$ & $\mathbb{Z}_{2}$ & 0 & $\mathbb{Z}_{2}$ & $Z$ \\
\hline$C_{4}^{1^{\prime}}$ & $E_{6} ; A_{7}^{\prime \prime}$ & $\mathrm{T}_{2} ; \mathbb{Z}_{2} \times \mathrm{T}_{1}$ & $A_{2}^{1}$ & $\mathbb{Z}_{2} \curlywedge \mathrm{SL}_{3}$ & $Z$ \\
\hline$C_{4}^{1^{\prime \prime}}$ & $A_{7}^{\prime}$ & $\mathrm{T}_{1}$ & $A_{1}^{4^{\prime}}$ & $\mathrm{SL}_{2}$ & $Z$ \\
\hline$D_{4}^{1}$ & $D_{4}$ & $\mathrm{~T}_{4}$ & $D_{4}^{1}$ & $\operatorname{Spin}_{8}$ & $\mathbb{S}_{3}<Z$ \\
\hline$D_{4}^{2^{\prime}}$ & $A_{7}^{\prime \prime} ; 2 D_{4}$ & $\mathbb{Z}_{2} \times \mathrm{T}_{1} ; \mathbb{V}_{4}$ & $A_{1}^{1}$ & $\mathbb{V}_{4} \times \mathrm{SL}_{2}$ & $\mathbb{S}_{4} \times Z^{\circ}$ \\
\hline$D_{4}^{2^{\prime \prime}}$ & $2 D_{4}$ & $\mathbb{V}_{4}$ & 0 & $\mathbb{V}_{4}$ & $\mathbb{S}_{4}$ \\
\hline$D_{4}^{2^{\prime \prime \prime}}$ & $A_{7}^{\prime} ; 2 D_{4}$ & $\mathrm{~T}_{1} ; \mathbb{V}_{4}$ & $T_{1}$ & $\mathbb{Z}_{2}<\mathrm{T}_{1}$ & $\mathbb{Z}_{2} \times Z$ \\
\hline$F_{4}^{1}$ & $E_{6}$ & $\mathrm{~T}_{2}$ & $G_{2}^{1}$ & $G_{2}$ & $Z$ \\
\hline$A_{5}^{1}$ & $A_{5}$ & $\mathrm{~T}_{3}$ & $A_{2}^{1}+A_{1}^{1}$ & $\mathrm{SL}_{3} \times \mathrm{SL}_{2}$ & $\mathbb{Z}_{2} \wedge Z$ \\
\hline$B_{5}^{1}$ & $D_{6}$ & $\mathrm{~T}_{2}$ & $B_{2}^{1}$ & $\mathrm{Sp}_{4}$ & $Z$ \\
\hline$D_{5}^{1}$ & $D_{5}$ & $\mathrm{~T}_{3}$ & $A_{3}^{1}$ & $\mathrm{SL}_{4}$ & $\mathbb{Z}_{2} \wedge Z$ \\
\hline$A_{6}^{1}$ & $A_{6}$ & $\mathrm{~T}_{2}$ & $A_{1}^{1}+T_{1}$ & $\mathrm{SL}_{2} \cdot \mathrm{T}_{1}$ & $\mathbb{Z}_{2} \wedge Z$ \\
\hline$D_{6}^{1}$ & $D_{6}$ & $\mathrm{~T}_{2}$ & $2 A_{1}^{1}$ & $\mathrm{SL}_{2}^{2}$ & $\mathbb{Z}_{2} \wedge Z$ \\
\hline$E_{6}^{1}$ & $E_{6}$ & $\mathrm{~T}_{2}$ & $A_{2}^{1}$ & $\mathrm{SL}_{3}$ & $\mathbb{Z}_{2} \wedge Z$ \\
\hline
\end{tabular}


TABLE $14 . G=E_{8}$ (continued)

\begin{tabular}{|c|c|c|c|c|c|}
\hline $\mathfrak{h}$ & $\mathcal{R}(\mathfrak{h})^{S}$ & $\mathcal{D}(\mathfrak{h})$ & $\mathfrak{z}$ & $Z$ & $N$ \\
\hline \hline$A_{7}^{1^{\prime}}$ & $A_{7}^{\prime \prime}$ & $\mathbb{Z}_{2} \times \mathrm{T}_{1}$ & $A_{1}^{1}$ & $\mathbb{Z}_{2} \times \mathrm{SL}_{2}$ & $\mathbb{Z}_{2} \times Z$ \\
\hline$A_{7}^{1^{\prime \prime}}$ & $A_{7}^{\prime}$ & $\mathrm{T}_{1}$ & $T_{1}$ & $\mathrm{~T}_{1}$ & $\mathbb{Z}_{2} \curlywedge Z$ \\
\hline$B_{7}^{1}$ & $D_{8}$ & $\mathbb{Z}_{2}$ & 0 & $\mathbb{Z}_{2}$ & $Z$ \\
\hline$D_{7}^{1}$ & $D_{7}$ & $\mathrm{~T}_{1}$ & $\mathrm{~T}_{1}$ & $\mathrm{~T}_{1}$ & $\mathbb{Z}_{2} \curlywedge Z$ \\
\hline$E_{7}^{1}$ & $E_{7}$ & $\mathrm{~T}_{1}$ & $A_{1}^{1}$ & $\mathrm{SL}_{2}$ & $Z$ \\
\hline$A_{8}^{1}$ & $A_{8}$ & $\mathbb{Z}_{3}$ & 0 & $\mathbb{Z}_{3}$ & $\mathbb{S}_{3}$ \\
\hline$D_{8}^{1}$ & $D_{8}$ & $\mathbb{Z}_{2}$ & 0 & $\mathbb{Z}_{2}$ & $Z$ \\
\hline
\end{tabular}

\section{REFERENCES}

[1] A. V. Alekseevskiŭ, Groups of components of centralizers of unipotent elements in semisimple algebraic groups, Trudy Tbilis. Mat. Inst. Razmadze 62 (1979), 5-27. (Russian) MR0557505 (81k:20063)

[2] È. B. Vinberg, The Weyl group of a graded Lie algebra, Izv. Akad. Nauk SSSR Ser. Mat. 40 (1976), 488-526; English transl. in Math. USSR-Izv. 10 (1977), 463-495. MR0430168 (55:3175)

[3] È. B. Vinberg and A. L. Onishchik, Seminar on Lie groups and algebraic groups, URSS, Moscow, 1995; English transl. of 1st ed., Springer-Verlag, Berlin, 1990. MR.1403378(97d:22001) MR.1064110 (91g:22001)

[4] Doan Kuin', The Poincaré polynomials of compact homogeneous Riemannian spaces with irreducible stationary group, Trudy Sem. Vector Tensor Anal. 14 (1968), 33-93. (Russian) MR0274665(43:427)

[5] E. B. Dynkin, Semisimple subalgebras of semisimple Lie algebras, Mat. Sb. 30 (1952), 349-462. (Russian) MR0047629 (13:904c)

[6] V. G. Kac, Automorphisms of finite order of semisimple Lie algebras, Funkts. Anal. Prilozh. 3, no. 3 (1969), 94-96; English transl., Funct. Anal. Appl. 3 (1969), 252-254. MR0251091 (40:4322)

[7] A. I. Mal'tsev, On semisimple subgroups of Lie groups, Izv. Akad. Nauk SSSR, Ser. Mat. 8, no. 4 (1944) 143-174. (Russian) MR0011303 (6:146b)

[8] I. V. Losev, On invariants of a set of elements of a semisimple Lie algebra, submitted to J. Lie Theory.

[9] M. W. Liebeck and G. M. Seitz, Reductive subgroups of exceptional algebraic groups, Mem. Amer. Math. Soc. vol. 121, no. 580, Amer. Math. Soc., Providence, RI, 1996. MR.1329942 (96i:20059)

[10] W. G. McKay and J. Patera, Tables of dimensions, indices, and branching rules for representations of simple Lie algebras, Lecture Notes in Pure and Appl. Math. vol. 69, Marcel Dekker, New York, 1981. MR0604363 (82i:17008)

Mechanics and Mathematics Department, Moscow State University, Leninskie Gory, MosCOW, GSP-2, 119992, Russia

E-mail address: andrei_msu@mail.ru

Translated by E. KHUKHRO 